CfPA-94-Th-55; UTAP-193

\title{
TOWARD UNDERSTANDING CMB ANISOTROPIES AND THEIR IMPLICATIONS ${ }^{\dagger}$
}

\author{
Wayne $\mathrm{Hu}^{1}$ and Naoshi Sugiyama ${ }^{1,2}$ \\ ${ }^{1}$ Departments of Astronomy and Physics \\ and Center for Particle Astrophysics \\ University of California, Berkeley, California 94720 \\ ${ }^{2}$ Department of Physics, Faculty of Science \\ The University of Tokyo, Tokyo, 113, Japan
}

\begin{abstract}
Working toward a model independent understanding of cosmic microwave background (CMB) anisotropies and their significance, we undertake a comprehensive and self-contained study of scalar perturbation theory. Initial conditions, evolution, thermal history, matter content, background dynamics, and geometry all play a role in determining the anisotropy. By employing analytic techniques to illuminate the numerical results, we are able to separate and identify each contribution. We thus bring out the nature of the total Sachs-Wolfe effect, acoustic oscillations, diffusion damping, Doppler shifts, and reionization, as well as their particular manifestation in a critical, curvature, or cosmological constant dominated universe. By studying the full angular and spatial content of the resultant anisotropies, we isolate the signature of these effects from the dependence on initial conditions. Whereas structure in the Sachs-Wolfe anisotropy depends strongly on the underlying power spectra, the acoustic oscillations provide features which are nearly model independent. This may allow for future determination of the matter content of the universe as well as the adiabatic and/or isocurvature nature of the initial fluctuations.
\end{abstract}

PACS: 98.70.Vc, 98.80.Es, 98.80.Hw.

hu@pac1.berkeley.edu, sugiyama@pac2.berkeley.edu

\footnotetext{
$\dagger$ Submitted to PRD. October 1994.
} 
It is only with people who know about the useless,

That there is any point in talking about uses.

In all the immensity of heaven and earth, A man uses no more than is room for his feet.

Yet if in recognition we were to cut all else away,

Would it still be useful to man?

-Chuang-tzu

\section{Introduction}

With the steadily increasing number of cosmic microwave background (CMB) anisotropy experiments on various angular scales (see e.g. [1]), the empirical reconstruction of the process for structure formation in the universe will soon enter a new phase. For this task to succeed, the groundwork for understanding anisotropy formation must be firmly laid. While numerical studies of specific models abound, this ab initio black box approach is not well suited to the reconstruction problem. One must be able to distinguish between the effects of initial conditions, evolution, thermal history, matter content, background dynamics, and geometry. With the goal of shedding light on the model independent physical mechanisms involved in anisotropy formation, we have undertaken a comprehensive and self-contained study of the scalar perturbations which give rise to large scale structure in the universe.

Of the two general classes of scalar perturbations, the isocurvature mode is by far the less well studied. A rich structure of anisotropies under the baryon isocurvature scenario is unveiled by generalizing the original model proposed by Peebles [2] to arbitrary thermal histories [3,4]. Yet even the familiar adiabatic case holds novel features if one steps beyond the standard $\Omega_{0}=1$ Harrison-Zel'dovich cold dark matter (CDM) model [5]. In this paper, we extend the highly accurate analytic tools developed for this standard CDM model [6] to the general case of arbitrary initial conditions, thermal history, and background dynamics. By employing these methods to illuminate the numerical results, we examine the physical mechanism behind the evolution of isocurvature and adiabatic fluctuations in an $\Omega_{0}=1$, open, or cosmological constant dominated universe, allowing for possible late or partial reionization. Focusing on the physical interpretation rather than specific model dependent results, we explore the possibilities that these as yet undetermined quantities leave open.

In $\S I I$, we discuss the gauge invariant perturbation equations and their general implications. Unlike most previous analytic treatments, e.g. $[7,8]$, we take a multifluid approach to realistically describe the evolution of each component. Superhorizon evolution, analyzed in $\S I I I$ brings out the differences between the isocurvature and adiabatic modes, including the gravitational redshift [9] and curvature effects [10]. Further discussion of open universe peculiarities may be found in Appendix A, and commonly used relations in Appendix B. As shown in $\S \mathrm{IV}$, intermediate scale perturbations in the photon-baryon fluid evolve as an oscillator in the potential well created by the total density perturbations. This leads to the characteristic oscillatory "Doppler" peak structure in the CMB at recombination for both modes [11]. Photon diffusion however erases these acoustic oscillations at small scales [12]. This is especially important in reionized scenarios, considered in $\S \mathrm{V}$, where last scattering is delayed and the diffusion length grows to be nearly the horizon size at last scattering. In this case, degree scale anisotropies can be dominated by the Doppler effect from scattering off electrons [13], which at late times are released from Compton drag. 
Putting these results together in $\S \mathrm{VI}$, we examine their implications for the observable quantities today. By analyzing the full matter and temperature transfer functions, we achieve separation of initial and evolutionary contributions. Robust features in the anisotropy are singled out as potentially useful for extracting information about the background cosmology. We conclude in $\S$ VII with some general comments on the present status of models given their predictions for CMB anisotropies.

\section{The Evolution Equations and Their Interpretation}

In this treatment, we assume scalar fluctuations about a background Friedmann-Robertson-Walker metric,

$$
d s^{2}=\left[\frac{a}{a_{0}}\right]^{2}\left(-d \eta^{2}+\gamma_{i j} d x^{i} d x^{j}\right),
$$

where $c=1, \gamma_{i j}$ is the 3-metric on a space of constant negative curvature, and $\eta \equiv \int\left(a_{0} / a\right) d t$ is the conformal time. Normalized to unity at matter-radiation equality, the scale factor $a$ evolves as $\dot{a} / a=H a / a_{0}$ where the overdot denotes a conformal time derivative and

$$
H^{2}=\left(\frac{a_{0}}{a}\right)^{4} \frac{1+a}{1+a_{0}} \Omega_{0} H_{0}^{2}-\left(\frac{a_{0}}{a}\right)^{2} K+\frac{1}{3} \Lambda,
$$

is the Hubble parameter with $H_{0}=100 \mathrm{hm} \mathrm{s}^{-1} \mathrm{Mpc}^{-1}$ as its value today. For spatially flat models, the curvature parameter $K=-H_{0}^{2}\left(1-\Omega_{0}-\Omega_{\Lambda}\right)$ goes zero, where the vacuum density is related to the cosmological constant by $\Omega_{\Lambda}=\Lambda / 3 H_{0}^{2}$.

Perturbations around these background quantities may be represented in various ways under gauge invariant theory $[14,15,16]$. Although all are gauge invariant, they reduce to ordinary perturbation quantities for different choices of hypersurface slicing [17]. This flexibility in the gauge invariant scheme allows us to simplify the physical interpretation. For temperature perturbations, we choose shear free Newtonian slicing, since on large scales, they are determined by gravitational redshifts from the Newtonian potential. Unfortunately, this choice does not clearly bring out the evolution of energy density perturbations, which is

best studied in the total matter rest frame representation. To avoid confusion, we will only employ photon and neutrino temperature perturbations, $\Theta=\Delta T_{\gamma} / T_{\gamma}$ and $N=\Delta T_{\nu} / T_{\nu}$, in the Newtonian representation, and energy density fluctuations, e.g. $\Delta_{\gamma}=\delta \rho_{\gamma} / \rho_{\gamma}$ and $\Delta_{\nu}=\delta \rho_{\nu} / \rho_{\nu}$, in the total matter rest frame representation.

\section{A. The Photon and Neutrino Boltzmann Equations}

The full linearized Boltzmann equation for the evolution of the Newtonian photon temperature perturbations $\Theta(\eta, \mathbf{x}, \gamma)$, is given by $[8]$

$$
\begin{aligned}
\frac{d}{d \eta}(\Theta+\Psi) & \equiv \dot{\Theta}+\dot{\Psi}+\dot{x}^{i} \frac{\partial}{\partial x^{i}}(\Theta+\Psi)+\dot{\gamma}^{i} \frac{\partial}{\partial \gamma^{i}}(\Theta+\Psi) \\
& =\dot{\Psi}-\dot{\Phi}+\dot{\tau}\left(\Theta_{0}-\Theta+\gamma_{i} v_{b}^{i}+\frac{1}{16} \gamma_{i} \gamma_{j} \Pi_{\gamma}^{i j}\right),
\end{aligned}
$$

where $\mathbf{v}_{b}$ is the baryon velocity $(c=1), \gamma_{i}\left(=\dot{x}_{i}\right)$ are the direction cosines of the photon momentum, $\Theta_{0}$ is the isotropic component of $\Theta$, and the anisotropic stress perturbation for the photons $\Pi_{\gamma}^{i j}$ is defined explicitly in Appendix A from the quadrupole moment of $\Theta$. The last term in equation (3) accounts for Compton scattering, where $\dot{\tau}=x_{e} n_{e} \sigma_{T} a / a_{0}$ is the differential optical depth, with $x_{e}$ the ionization fraction, $n_{e}$ the 
electron number density, and $\sigma_{T}$ the Thomson cross section. The gauge invariant metric perturbations are $\Psi$, the Newtonian potential and $\Phi$, the perturbation to the intrinsic spatial curvature, which are related to the density perturbation through a generalized Poisson equation in $\S$ IIC. We will commonly refer to both $\Phi$ and $\Psi$ as gravitational potentials.

If the potentials are static and Compton scattering is ineffective, equation (3) implies $\Theta+\Psi$ is a conserved quantity. This merely represents what we call the ordinary Sachs-Wolfe (SW) effect: a photon experiences a fractional redshift of $\Psi$ climbing out of a $\Psi<0$ potential well. The effective temperature perturbation accounting for this shift is therefore $\Theta+\Psi$. If $\Psi$ changes, the corresponding gravitational redshift of course follows suit. Changes in $\Phi$ also affect the photons through time dilation. Since these effects accumulate along the geodesics, we call the combination the integrated Sachs-Wolfe (ISW) effect. The total contribution, derived by Sachs and Wolfe [9], is a combination of SW and ISW effects and completely describes the effect of gravitational redshift on the photons.

In open universes, the $\dot{\gamma}_{i}$ term in equation (3) does not vanish due to the curving of geodesics. Although this would seem to complicate matters, its effect on equation (3) is easy to interpret and compute, once we decompose the fluctuation into its normal modes. Plane wave perturbations $Q=\exp (i \mathbf{k} \cdot \mathbf{x})$, appropriate for a flat geometry, must be replaced with the eigenfunctions of the Laplacian for an open geometry $[18,19,20]$ :

$$
\nabla^{2} Q \equiv \gamma^{i j} Q_{\mid i j}=-k^{2} Q
$$

where "l" denotes a covariant derivative on the 3 -space. Since the eigenfunctions are complete for $k \geq \sqrt{-K}$ one often introduces the auxiliary variable $\tilde{k}^{2}=k^{2}+K$. The subtle question of whether $2 \pi / k$ or $2 \pi / \tilde{k}$ should be considered as the "physical" wavelength of the mode is examined further in Appendix A.

Since each eigenmode evolves independently in linear theory, it is sufficient to consider temperature perturbations to exist in a single $k$-mode, ${ }^{*}$ which can be decomposed into angular moments as $[10,21]$

$$
\Theta(\eta, \mathbf{x}, \gamma)=\sum_{\ell=0}^{\infty} \Theta_{\ell}(\eta) G_{\ell}(\mathbf{x}, \gamma)
$$

Here the angular functions $G_{\ell}$ are defined in Appendix A such that they reduce to $G_{\ell}=(-i)^{\ell} \exp (i \mathbf{k}$. $\mathbf{x}) P_{\ell}(\mathbf{k} \cdot \gamma)$ in the flat space limit, where $P_{\ell}$ is an ordinary Legendre polynomial.

We can now write equation (3) in the standard hierarchy of coupled equations for the $\ell$-modes:

$$
\begin{aligned}
& \dot{\Theta}_{0}=-\frac{k}{3} \Theta_{1}-\dot{\Phi} \\
& \dot{\Theta}_{1}=k\left[\Theta_{0}+\Psi-\frac{2}{5}\left(1-\frac{3 K}{k^{2}}\right) \Theta_{2}\right]-\dot{\tau}\left(\Theta_{1}-V_{b}\right), \\
& \dot{\Theta}_{2}=k\left[\frac{2}{3} \Theta_{1}-\frac{3}{7}\left(1-\frac{8 K}{k^{2}}\right) \Theta_{3}\right]-\frac{9}{10} \dot{\tau} \Theta_{2}, \\
& \dot{\Theta}_{\ell}=k\left[\frac{\ell}{2 \ell-1} \Theta_{\ell-1}-\frac{\ell+1}{2 \ell+3}\left(1-\ell(\ell+2) \frac{K}{k^{2}}\right) \Theta_{\ell+1}\right]-\dot{\tau} \Theta_{\ell}, \quad(\ell>2)
\end{aligned}
$$

* As usual, the general case can be recovered by summing a power spectrum of these independent $k$-modes. It should also be noted that all perturbation amplitudes such as $\Theta_{\ell}$ have an implicit $k$-dependence. However when discussing the evolution of a single $k$-mode, we drop the index for brevity. After this section, no real space perturbation variables are employed. 
where $\gamma_{i} v_{b}^{i}=V_{b} G_{1}=V_{b}(-k)^{-1} \gamma_{i} Q_{\mid i}$, and we have made the replacements such as $\Psi(\eta, \mathbf{x})=\Psi(\eta) G_{0}(\mathbf{x})=$ $\Psi(\eta) Q(\mathbf{x})$ here and below. By analogy to equation (6), we can immediately write down the corresponding Boltzmann equation for (massless) neutrino temperature perturbations $N(\eta, \mathbf{x}, \gamma)$ by making the replacements $\Theta_{\ell} \rightarrow N_{\ell}, \dot{\tau} \rightarrow 0$, in equation (6). This is sufficient since neutrino decoupling occurs before any scale of interest enters the horizon.

\section{B. CMB Anisotropies}

Although this Newtonian representation of the Boltzmann equation (6) may cause stability problems for its numerical solution [22], it serves to bring out the physics of anisotropies quite well. First, scattering tends to isotropize the photons in the electron rest frame, leaving anisotropies only in the unscattered fraction: for $\ell>2, \Theta_{\ell} \propto \exp (-\tau)$, whereas $\Theta_{2} \propto \exp (-9 \tau / 10)$ due to the angular dependence of Compton scattering. Isotropy also requires $V_{\gamma} \equiv \Theta_{1}=V_{b}$. Even so, the dipole suffers from gravitational infall due to $\Psi$, i.e. the SW effect. On the other hand, the ISW effect provides a source to the monopole.

Since the density of free electrons decreases either due to recombination, or if the universe is reionized, to the expansion, the CMB eventually ceases to scatter when the optical depth to the present from Compton scattering drops to $\int_{\eta_{*}}^{\eta_{0}} \dot{\tau} d \eta=1$. Under the standard recombination scenario, this occurs at $z_{*} \simeq 1000$, whereas for reionized models it is delayed until

$$
z_{*} \simeq 30\left(\frac{\Omega_{0} h^{2}}{0.1}\right)^{1 / 3}\left(\frac{0.05}{x_{e} \Omega_{b} h^{2}}\right)^{2 / 3},
$$

if last scattering occurs before curvature or $\Lambda$ domination.

After $z_{*}$, the photons effectively free stream to form anisotropies. On the last scattering surface, the photon distribution may be locally isotropic while still possessing inhomogeneities, i.e. hot and cold spots, which will be observed as anisotropies on the sky today. Free streaming transfers fluctuations to high multipoles, as the $\ell$-mode coupling of equation (6) shows. Consequently, in the absence of sources, the monopole collisionlessly damps. For superhorizon scales $k \eta \ll 1$, the photons can only travel a small fraction of a wavelength, and thus the fluctuations remain in the monopole. This is reflected in the $k$-dependence of this $\ell$-mode coupling. If there is subsequent reionization, superhorizon sized fluctuations will consequently not damp by isotropization.

Due to the more rapid deviation of geodesics, a given length scale will correspond to a smaller angle in an open universe than a flat one. Thus the only effect of negative spatial curvature in equation (6) is to speed the transfer of power to higher multipoles. Its effect is noticeable if the angular scale $\theta \sim \ell^{-1}$ is less than the ratio of the physical scale to the curvature radius $\sqrt{-K} / k$. One peculiarity arises however. Even for the lowest eigenmode, $k=\sqrt{-K}$ or $\tilde{k}=0$, the $\ell$-mode coupling in equation (6) does not vanish. Unlike the flat case, this "infinite wavelength" mode suffers free streaming damping of low order multipoles, once the horizon becomes larger than the curvature radius $\eta \sqrt{-K} \gtrsim 1$. The physical origin of this effect is discussed further in $§$ VIB and Appendix A.

Finally, let us state some useful relations. As discussed in Appendix A, the total anisotropy is

$$
\begin{aligned}
\frac{2 \ell+1}{4 \pi} C_{\ell} & =\frac{V}{2 \pi^{2}} \int \frac{d \tilde{k}}{\tilde{k}} \frac{M_{\ell}}{2 \ell+1} \tilde{k}^{3}\left|\Theta_{\ell}\right|^{2} \\
& =\frac{V}{2 \pi^{2}} \int_{\sqrt{-K}}^{\infty} \frac{d k}{k} \frac{M_{\ell}}{2 \ell+1}\left(1+K / k^{2}\right)^{1 / 2} k^{3}\left|\Theta_{\ell}\right|^{2},
\end{aligned}
$$


where the ensemble average anisotropy predicted for an experiment with window function $W_{\ell}$ is $(\Delta T / T)^{2}=$ $\sum(2 \ell+1) W_{\ell} C_{\ell} / 4 \pi$ with $\Theta_{\ell}$ evaluated at present. Here $M_{\ell}=\left(\tilde{k}^{2}-K\right) \ldots\left(\tilde{k}^{2}-K \ell^{2}\right) /\left(\tilde{k}^{2}-K\right)^{\ell}$ and reduces to unity in the flat space limit. This implies that the contribution to the anisotropy per logarithmic $k$ and $\ell$ interval is

$$
\left(\frac{\Delta T}{T}\right)_{\ell k}^{2} \equiv \frac{\ell M_{\ell}}{2 \ell+1}\left(1+K / k^{2}\right)^{1 / 2} k^{3} V\left|\Theta_{\ell}\right|^{2} .
$$

We can also sum in $\ell$ to obtain

$$
|\Theta+\Psi|_{r m s}^{2} \equiv\left|\Theta_{0}+\Psi\right|^{2}+\sum_{\ell=1}^{\infty} \frac{M_{\ell}}{2 \ell+1}\left|\Theta_{\ell}\right|^{2},
$$

which measures the total power in a single $k$-mode. Since fluctuations are merely transferred to high multipoles by free streaming, this quantity is conserved if $\dot{\Phi}=\dot{\Psi}=\dot{\tau}=0$, as is evident from equation (3).

\section{Gravitational Potentials}

We of course have to define the gravitational potentials $\Phi$ and $\Psi$ in order to complete the Boltzmann equation (6). It is useful to introduce the following gauge invariant variables: the total density perturbation in the matter rest frame $\rho \Delta_{T}=\sum_{i} \rho_{i} \Delta_{i}$, where the sum runs over all the species present and the density perturbations are related to the temperature perturbations by

$$
\begin{aligned}
& \Delta_{\gamma}=4 \Theta_{0}+4 \frac{\dot{a}}{a} \frac{V_{T}}{k}, \\
& \Delta_{\nu}=4 N_{0}+4 \frac{\dot{a}}{a} \frac{V_{T}}{k} ;
\end{aligned}
$$

the total matter velocity $(\rho+p) V_{T}=\sum_{i}\left(\rho_{i}+p_{i}\right) V_{i}$, where $p$ is the pressure; and the anisotropic stress $p \Pi=\sum_{i} p_{i} \Pi_{i}$, where contributions come essentially from the radiation quadrupoles

$$
\Pi_{\gamma}=\frac{12}{5} \Theta_{2}, \quad \Pi_{\nu}=\frac{12}{5} N_{2} .
$$

Employing the Einstein equations, we may now write the generalized Poisson equation as

$$
\begin{aligned}
\Phi & =\frac{4 \pi G}{k^{2}-3 K}\left(\frac{a}{a_{0}}\right)^{2} \rho \Delta_{T}, \\
\Phi+\Psi & =-\frac{8 \pi G}{k^{2}}\left(\frac{a}{a_{0}}\right)^{2} p \Pi .
\end{aligned}
$$

As discussed above, scattering suppresses anisotropies such that $\Theta_{2} \simeq 0$, and for perturbations larger than the horizon scale, $N_{2} \ll N_{0}$. Moreover, in the matter dominated regime, the pressure itself is negligible $p \ll \rho$. The $p \Pi$ anisotropic stress term can thus be ignored as a first approximation, implying $\Phi \simeq-\Psi$. 


\section{Matter Components}

The baryons evolve under the generalized baryon continuity and Euler equations

$$
\begin{aligned}
\dot{\Delta}_{b} & =-k\left(V_{b}-V_{\gamma}\right)+\frac{3}{4} \dot{\Delta}_{\gamma}, \\
\dot{V}_{b} & =-\frac{\dot{a}}{a} V_{b}+k \Psi+\dot{\tau}\left(V_{\gamma}-V_{b}\right) / R,
\end{aligned}
$$

where $R \equiv 3 \rho_{b} / 4 \rho_{\gamma}$ is the scale factor normalized to $3 / 4$ at photon-baryon equality. Again if a collisionless non-relativistic particle were present, e.g. CDM or compact baryonic objects [23,24], its evolution would be obtained by setting $\dot{\tau}=0$.

Well inside the horizon, equation (6) and (11) imply that the photons satisfy a separate continuity equation $\dot{\Delta}_{\gamma}=-4 / 3 k V_{\gamma}$, which reduces the first baryon equation to the familiar form $\dot{\Delta}_{b}=-k V_{b}$. The baryon velocity decays due to the expansion and has a source term from infall into gravitational wells. Thus the only effect of the decoupled components is through this potential term.

Early on scattering makes $V_{b}=V_{\gamma}$, which shows that the photons and baryons evolve adiabatically $\dot{\Delta}_{\gamma}=4_{3} \dot{\Delta}_{b}$, regardless of whether the initial conditions are adiabatic or isocurvature. Yet even if the universe remains fully ionized to the present, the baryons will eventually decouple from the photons, because $\dot{\tau} / R=4 /{ }_{3} \dot{\tau}\left(\rho_{\gamma} / \rho_{b}\right)$ goes to zero in the matter dominated limit. Since $\tau \propto \Omega_{b}$, this epoch is independent of $\Omega_{b}$. The Compton drag on an individual baryon does not depend on the total number of baryons. In fact, equation (14) and the Poisson (13) equations show that the drag term $\propto V_{b}$ comes to dominate over the

gravitational infall term $\propto k \Psi$ at redshifts above $z \sim 200\left(\Omega_{0} h^{2}\right)^{1 / 5} x_{e}^{-2 / 5}$. Thus all modes are released from Compton drag at the same time, which we take to be

$$
z_{d}=160\left(\Omega_{0} h^{2}\right)^{1 / 5} x_{e}^{-2 / 5}
$$

defined as the epoch when fluctuations effectively join the growing mode of pressureless linear theory (see $\S \mathrm{VA})$.

It is important to realize that the drag and the last scattering redshift are generally not equal. The photons decouple from the baryons before the baryons decouple from the photons in the standard recombination scenario. Typically the opposite occurs in reionized scenarios resulting in quite different anisotropies for the two cases (see $\S I V$ and V).

We now possess all the machinery necessary to describe the evolution of perturbations. Numerical solutions, based on Sugiyama \& Gouda [25], are presented in the following sections. However, to shed light on these solutions, we also apply analytic techniques in the single fluid (§III), photon-baryon fluid (§IV), and diffusive limits $(\S \mathrm{V})$. 


\section{Large Scale Evolution: Single Fluid Approximation}

Since no causal process such as free streaming or diffusion can separate the components, all fluid velocities are equal above the horizon. We can thus describe the coupled multi-component system as a single fluid, defined by the total matter variables, whose behavior does not even depend on the ionization history. Its evolution is determined by combining the equations for the various species, assumed to be either fully relativistic or non-relativistic, i.e. equations (6) and (14) with their decoupled variants,

$$
\begin{aligned}
\dot{\Delta}_{T}-3 w \frac{\dot{a}}{a} \Delta_{T} & =-\left(1-\frac{3 K}{k^{2}}\right)(1+w) k V_{T}-2\left(1-\frac{3 K}{k^{2}}\right) \frac{\dot{a}}{a} w \Pi, \\
\dot{V}_{T}+\frac{\dot{a}}{a} V_{T} & =\frac{4}{3} \frac{w}{(1+w)^{2}} k\left[\Delta_{T}-(1-3 w) S\right]+k \Psi-\frac{2}{3} k\left(1-\frac{3 K}{k^{2}}\right) \frac{w}{1+w} \Pi,
\end{aligned}
$$

where $w=p / \rho$ and $S \equiv \Delta_{m}-3 / 4 \Delta_{r}$, with $\Delta_{m}$ and $\Delta_{r}$ being the perturbations in the matter and radiation energy densities respectively. As we shall see, $S$ can be interpreted as an entropy fluctuation. In the evolution equation for $V_{T}$, infall due to the potential $\Psi$ is countered by the pressure term $\Delta_{T}$ at small scales. The two are balanced at the Jeans scale. In this section, we solve the evolution equations neglecting pressure and anisotropic stress as is appropriate for large scales.

\section{A. Initial Conditions and the General Solution}

The distinction between adiabatic and isocurvature scenarios lies in the entropy term $S$ of equation (16). Its evolution is given by the matter continuity equation (14), i.e. $\dot{S}=k\left(V_{r}-V_{m}\right)$, where the matter and radiation velocities are defined in a manner analogous to $V_{T}$ [see equation (11)]. Since all components have the same velocity, $S$ is a constant before the mode enters the horizon and, if it is present, must have been established at the initial conditions.

Under this $\dot{S}=0$ assumption, we show in Appendix B that the general growing solution of equation (16) is

$$
\Delta_{T}=C_{A} U_{A}+C_{I} U_{I}
$$

where the $C$ 's are fixed by the initial conditions, and we have neglected anisotropic stress. Although $S$ is a constant above the horizon, we will define $C_{I} \equiv S(0)$, in anticipation of horizon crossing. As we shall see

in the next section, the evolutionary factors $U_{A}$ and $U_{I}$ take simple asymptotic forms. However to preserve generality, we give the complete expressions here:

$$
\begin{aligned}
U_{A} & =\left[D^{3}+\frac{2}{9} D^{2}-\frac{8}{9} D-\frac{16}{9}+\frac{16}{9} \sqrt{D+1}\right] \frac{1}{D(D+1)}, \\
U_{I} & =\frac{4}{15}\left(\frac{k}{k_{e q}}\right)^{2}\left(1-\frac{3 K}{k^{2}}\right) \frac{3 D^{2}+22 D+24+4(4+3 D)(1+D)^{1 / 2}}{(1+D)(4+3 D)\left[1+(1+D)^{1 / 2}\right]^{4}} D^{3}
\end{aligned}
$$

respectively, where $k_{e q} \equiv(a H)_{e q} / a_{0}=\sqrt{2}\left(a_{0} \Omega_{0} H_{0}^{2}\right)^{1 / 2}$ corresponds to the scale which passes the horizon at equality, and we have assumed $\Pi=0$. The factor $D(a)$ accounts for pressureless growth

$$
D=\frac{5}{4} k_{e q}^{2} \tilde{H} \int \frac{d a / a_{0}}{\left(\tilde{H} a / a_{0}\right)^{3}},
$$

where $\tilde{H}$ is obtained from the Hubble parameter by ignoring the radiation

$$
\tilde{H}^{2}=\left(\frac{a_{0}}{a}\right)^{3} \Omega_{0} H_{0}^{2}-\left(\frac{a_{0}}{a}\right)^{2} K+\frac{1}{3} \Lambda .
$$


We assume curvature and $\Lambda$ dynamical contributions are only important well after equality $a \gg 1$. Curvature dominates over matter at $a / a_{0}>\Omega_{0} /\left(1-\Omega_{0}-\Omega_{\Lambda}\right)$, whereas $\Lambda$ dominates over matter at $a / a_{0}>\left(\Omega_{0} / \Omega_{\Lambda}\right)^{1 / 3}$ and over curvature at $a / a_{0}>\left[\left(1-\Omega_{0}-\Omega_{\Lambda}\right) / \Omega_{\Lambda}\right]^{1 / 2}$. Although we will usually only consider $\Lambda$ models which are flat, these solutions are applicable to the general case. Before either curvature or $\Lambda$ domination, $D=a$; afterwards, it goes to a constant. Moreover if $a \gg 1, \Delta_{T} \propto D$ regardless of scale and initial conditions as discussed below.

Whereas adiabatic scenarios begin with $C_{I}=0$ and finite $C_{A}$, isocurvature universes have $C_{I} \neq 0$. If only baryons, photons and neutrinos are present, as in the case of the baryonic models, $S$ can be broken down into

$$
\begin{aligned}
S & =f_{\nu}\left(\Delta_{b}-\frac{3}{4} \Delta_{\nu}\right)+\left(1-f_{\nu}\right)\left(\Delta_{b}-\frac{3}{4} \Delta_{\gamma}\right) \\
& =\left(1-f_{\nu}\right) S_{b \gamma}+f_{\nu} S_{b \nu}
\end{aligned}
$$

with an obvious generalization to the case of an additional decoupled non-relativistic component. Here the neutrino fraction $f_{\nu}=\rho_{\nu} /\left(\rho_{\nu}+\rho_{\gamma}\right)$ is time independent after electron-positron annihilation, implying $f_{\nu}=0.405$ for three massless neutrinos and the standard thermal history. Since $S_{b \gamma} \equiv \Delta_{b}-3 /{ }_{4} \Delta_{\gamma}=\delta\left(n_{b} / n_{\gamma}\right)$, and likewise for the neutrinos, $S_{b \gamma}$ and $S_{b \nu}$ represent perturbations to the baryon to photon and baryon to neutrino number densities respectively. This in fact justifies our use of the term "entropy" fluctuation to describe $S$. Notice that $\dot{S}=0$ then has an obvious interpretation: since the components cannot separate above the horizon, the particle number ratios must remain constant.

Furthermore isocurvature conditions allow no initial curvature perturbations as the name implies. Thus the gravitational potentials $\Psi$ and $\Phi$ vanish at the initial epoch, implying $C_{A}=0$. If the perturbations were formed in the radiation dominated epoch, they must be placed in the baryons only. In this case, $S_{b \gamma}=S_{b \nu}=S$ initially, which we will hereafter adopt. The generalization to the case where $S_{b \gamma} \neq S_{b \nu}$ is straightforward. Note that any arbitrary mixture of adiabatic and isocurvature modes is also covered by equation (17).

\section{B. The Evolution of the Fluid Components}

Now let us consider the implications and interpretation of the general solution (17). The results for the adiabatic mode are extremely simple. When the universe is dominated by radiation (RD), matter (MD), curvature $(\mathrm{CD})$ or the cosmological constant $(\Lambda \mathrm{D})$, the total density fluctuation takes the form

$$
\Delta_{T} / C_{A}= \begin{cases}\frac{10}{9} a^{2} & \mathrm{RD} \\ a & \mathrm{MD} \\ D . & \mathrm{CD} / \Lambda \mathrm{D}\end{cases}
$$

Moreover since $S=0$, the components evolve together $\Delta_{b}=\Delta_{c}=3 / 4 \Delta_{\gamma}=3 /{ }_{4} \Delta_{\nu}$ where $\Delta_{c}$ is any decoupled non-relativistic component (e.g. CDM). The velocity and potential are given by

$$
\begin{aligned}
V_{T} / C_{A}= \begin{cases}-\frac{5 \sqrt{2}}{12}\left(k_{e q} / k\right)\left(1-3 K / k^{2}\right)^{-1} a & \mathrm{RD} \\
-\frac{\sqrt{2}}{2}\left(k_{e q} / k\right)\left(1-3 K / k^{2}\right)^{-1} a^{1 / 2} & \mathrm{MD} \\
-\left(1-3 K / k^{2}\right)^{-1} \dot{D} / k & \mathrm{CD} / \Lambda \mathrm{D}\end{cases} \\
\Psi / C_{A}= \begin{cases}-\frac{5}{6}\left(k_{e q} / k\right)^{2}\left(1-3 K / k^{2}\right)^{-1} & \mathrm{RD} \\
-\frac{3}{4}\left(k_{e q} / k\right)^{2}\left(1-3 K / k^{2}\right)^{-1} & \mathrm{MD} \\
-\frac{3}{4}\left(k_{e q} / k\right)^{2}\left(1-3 K / k^{2}\right)^{-1} D / a . & \mathrm{CD} / \Lambda \mathrm{D}\end{cases}
\end{aligned}
$$


Contrast this with the isocurvature evolution,

$$
\Delta_{T} / C_{I}= \begin{cases}\frac{1}{6}\left(k / k_{e q}\right)^{2}\left(1-3 K / k^{2}\right) a^{3} & \mathrm{RD} \\ \frac{4}{15}\left(k / k_{e q}\right)^{2}\left(1-3 K / k^{2}\right) a & \mathrm{MD} \\ \frac{4}{15}\left(k / k_{e q}\right)^{2}\left(1-3 K / k^{2}\right) D . & \mathrm{CD} / \Lambda \mathrm{D}\end{cases}
$$

From the definition of the entropy fluctuation $S$ (see also Appendix B), equation (18) implies that

$$
\Delta_{b} / C_{I}= \begin{cases}1-\frac{3}{4} a & \mathrm{RD} \\ \frac{4}{3}\left[a^{-1}+\frac{1}{5}\left(k / k_{e q}\right)^{2}\left(1-3 K / k^{2}\right) a\right] & \mathrm{MD} \\ \frac{4}{3}\left[a^{-1}+\frac{1}{5}\left(k / k_{e q}\right)^{2}\left(1-3 K / k^{2}\right) D\right], & \mathrm{CD} / \Lambda \mathrm{D}\end{cases}
$$

and

$$
\Delta_{\gamma} / C_{I}=\Delta_{\nu} / C_{I}= \begin{cases}-a & \mathrm{RD} \\ \frac{4}{3}\left[-1+\frac{4}{15}\left(k / k_{e q}\right)^{2}\left(1-3 K / k^{2}\right) a\right] & \mathrm{MD} \\ \frac{4}{3}\left[-1+\frac{4}{15}\left(k / k_{e q}\right)^{2}\left(1-3 K / k^{2}\right) D\right], & \mathrm{CD} / \Lambda \mathrm{D}\end{cases}
$$

for the baryon and radiation components. Lastly, the velocity and the potential also have simple asymptotic forms,

$$
\begin{aligned}
V_{T} / C_{I} & = \begin{cases}-\frac{\sqrt{2}}{8}\left(k / k_{e q}\right) a^{2} & \mathrm{RD} \\
-\frac{2 \sqrt{2}}{15}\left(k / k_{e q}\right) a^{1 / 2} & \mathrm{MD} \\
-\frac{4}{15}\left(k / k_{e q}\right) \dot{D} / k_{e q}, & \mathrm{CD} / \Lambda \mathrm{D}\end{cases} \\
\Psi / C_{I} & = \begin{cases}-\frac{1}{8} a & \mathrm{RD} \\
-\frac{1}{5} & \mathrm{MD} \\
-\frac{1}{5} D / a . & \mathrm{CD} / \Lambda \mathrm{D}\end{cases}
\end{aligned}
$$

Notice that unlike the adiabatic case $V_{T}$ and $\Psi$ have no explicit curvature dependence in this representation. Moreover, although these solutions omit radiation pressure and streaming, they are actually valid for the matter all the way to the present if horizon crossing occurs after the drag epoch (see Fig. 1).

Let us try to interpret these results physically. The isocurvature condition is satisfied by initially placing the fluctuations in the baryons $\Delta_{b}=C_{I}$ with $\Delta_{\gamma}=0$, so that $\Delta_{T}=0$. As the universe evolves however, the relative significance of the baryon fluctuation $\Delta_{b} \rho_{b} / \rho_{T}$ for the total density fluctuation $\Delta_{T}$ grows as $a$. To compensate, the photon and neutrino fluctuations grow to be equal and opposite $\Delta_{\gamma}=\Delta_{\nu}=-a C_{I}$. The tight coupling condition $\dot{\Delta}_{b}=3 / 4 \dot{\Delta}_{\gamma}$ implies then that the baryon fluctuation must also decrease so that $\Delta_{b}=(1-3 a / 4) C_{I}$. The presence of $\Delta_{\gamma}$ means that there is a gradient in the photon energy density. This gradient gives rise to a dipole $V_{\gamma}$ as the regions come into causal contact [see equation (6)], i.e. $V_{\gamma} \propto k \eta \Delta_{\gamma} \propto-k a^{2} C_{I}$. The same argument holds for the neutrinos. Constant entropy requires that the total fluid move with the photons and neutrinos $V_{T}=V_{\gamma}$, and thus infall, produced by the gradient in the velocity, yields a total density perturbation $\Delta_{T} \propto-k \eta\left(1-3 K / k^{2}\right) V_{T} \propto k^{2}\left(1-3 K / k^{2}\right) a^{3} C_{I}$ [see equation (16)]. This is one way of interpreting equation (18) and the fact that the entropy provides a source of total density fluctuations in the radiation dominated epoch [26].

A similar analysis applies for adiabatic fluctuations, which begin instead with finite potential $\Psi$. Infall implies $V_{T} \propto k \eta \Psi$, which then yields $\Delta_{T} \propto-k \eta V_{T} \propto-k^{2}\left(1-3 K / k^{2}\right) a^{2} \Psi$, thereby also keeping the potential constant. Compared to the adiabatic case, the isocurvature scenario predicts total density perturbations which are smaller by one factor of $a$ in the radiation dominated epoch as might be expected from cancellation.

After radiation domination both modes grow in pressureless linear theory $\Delta_{T} \propto D$ [c.f. equations (22) and (24)]. Whereas in the radiation dominated limit, the entropy term $S$ and the gravitational infall 
term $\Psi$ are comparable in equation (16), the entropy source is thereafter suppressed by $w=p / \rho$, making the isocurvature and adiabatic evolutions identical. Since growth is suppressed in open and $\Lambda$ dominated universes, the potential $\Psi$ decays which has interesting consequences for anisotropies as we shall now see.

\section{The Total Sachs-Wolfe Effect: Basics}

As noted in $\S I I B$, the SW effect causes the effective perturbation to be $\Theta+\Psi$ to account for the gravitational redshift. Subsequent changes in the potential of course alter the shift, an effect which is approximately doubled by the induced time dilation. This is the ISW contribution. To determine the net effect however, we must first derive the value of the intrinsic photon fluctuations $\Theta$. If $k \eta \ll 1$, the Boltzmann equation (6) reduces to the ISW effect

$$
\dot{\Theta}_{0}=-\dot{\Phi} \simeq \dot{\Psi}
$$

Here we have again assumed $\Pi=0$, which causes a $\sim 10 \%$ error. For corrections due to $\Pi$ see [6].

Since the isocurvature initial conditions satisfy $\Psi(0)=0=\Theta_{0}(0)$, this implies $\Theta_{0}(\eta)=\Psi(\eta)$. The effective superhorizon scale temperature perturbation for isocurvature fluctuations is therefore

$$
\Theta_{0}+\Psi=2 \Psi . \quad \text { (iso) }
$$

Inside potential wells, the ISW effect makes photons underdense so that the gravitational redshift adds to the temperature perturbation. This is a direct consequence of the feedback mechanism which generates the potentials (see $\S \mathrm{IIIB}$ ). Note however that in a low $\Omega_{0} h^{2}$ model with standard recombination, the potential may not reach its full matter dominated value equation (27) by last scattering (see Fig. 2).

For adiabatic perturbations, the nature of the growing mode $U_{G}$ [see equation (18)] fixes the initial

perturbations to be $\Theta_{0}(0)=-\frac{1}{2} \Psi(0)$, reflecting the fact that the photons are overdense inside the potential well. Although $U_{G}$ implies the potential is constant in both the matter and radiation dominated epoch, it changes to $\Psi(a)=\frac{9}{10} \Psi(0)$ through equality. The ISW effect then brings the photon temperature perturbation in the matter dominated epoch to $\Theta(\eta)=-\frac{2}{3} \Psi(\eta)$ and the effective superhorizon perturbation to (MD)

$$
\left[\Theta_{0}+\Psi\right]=\frac{1}{3} \Psi, \quad(\text { adi })
$$

which is the familiar Sachs-Wolfe result.

The above results are valid before last scattering at $a_{*}$. Again since last scattering often occurs before full matter domination, one should employ the full form $\left[\Theta_{0}+\Psi\right]\left(\eta_{*}\right) \simeq \Theta_{0}(0)+2 \Psi\left(\eta_{*}\right)-\Psi(0)$ instead of equations (27) and (30). After $a_{*}$, the photons climb out of the potential wells, leaving the quantity $\left[\Theta_{0}+\Psi\right]\left(\eta_{*}\right)$ to become anisotropies today. Additional contributions to the anisotropy may arise during free streaming again through the ISW effect. Since photon geodesics are radial in the absence of scattering, we may use the radial eigenfunctions of the Laplacian to solve for the free streaming behavior. These are denoted by $Q(\mathbf{x})=X_{\nu}^{\ell}(\chi) Y_{\ell}^{m}(\theta, \phi)$, where $-\nu^{2}=\tilde{k}^{2} / K=k^{2} / K+1$, and the radial distance normalized to the curvature radius is $\chi=\sqrt{-K} \eta$. The radial function $X_{\nu}^{\ell}(\chi)$ reduces to $j_{\ell}(k \eta)$ in the flat limit. For superhorizon scales at last scattering, the resultant anisotropies are (see Appendix A),

$$
\frac{\Theta_{\ell}(\eta)}{2 \ell+1}=\left[\Theta_{0}+\Psi\right]\left(\eta_{*}\right) X_{\nu}^{\ell}\left(\chi-\chi_{*}\right)+\int_{\eta_{*}}^{\eta}(\dot{\Psi}-\dot{\Phi}) X_{\nu}^{\ell}\left(\chi-\chi^{\prime}\right) d \eta^{\prime} .
$$


The right hand side represents the SW and ISW effects respectively. Since the potentials for both the adiabatic and isocurvature modes are constant in the matter dominated epoch, the ISW contribution is separated into two parts:

(a) The early ISW effect due to pressure growth suppression after horizon crossing in the radiation dominated epoch.

(b) The late ISW effect due to expansion growth suppression in the $\Lambda$ or curvature dominated epoch.

We shall now consider these effects in more detail.

\section{The Total Sachs-Wolfe Effect: Detailed Structure}

Equation (31) for the total Sachs-Wolfe effect predicts a rich structure of anisotropies for low $\Omega_{0}$ models [5]. However, to build intuition for equation (31), let us first consider the familiar adiabatic $\Omega_{0}=1$ model in which the ISW term represents only a small correction [6]. A given $k$-mode contributes maximum anisotropies to the angle that scale subtends on the sky at last scattering. In the $k-\ell$ plane, the anisotropy will have a sharp ridge corresponding to this correlation (see Fig. 3). Here we have plotted $(\Delta T / T)_{\ell k}^{2}$, the logarithmic contribution to the anisotropy in $k$ and $\ell$ defined by equation (9).* The pure spherical Bessel functions $j_{\ell}(x)$ show that the series of ridges and filamentary structures are due to the structure of the radial eigenfunctions themselves. Notice that the largest $k$-modes project onto the monopole and do not contribute to anisotropies.

Now let us move onto the more complicated $\Lambda$ and open cases. For $\Lambda$ models, the ISW term in equation (31) yields both early and late type contributions. As we shall see (see $\S \mathrm{IV}$ ), inside the horizon during the radiation dominated era, the potential decays due to pressure. This leads to a significant early ISW term which is projected onto a somewhat larger angle than the SW effect itself since it originates closer to the present. That this is present before $\Lambda$ domination is clear from Fig. 4a. Because the early ISW effect approaches its maximum of $[\Psi-\Phi]\left(\eta_{*}\right) \simeq 2 \Psi\left(\eta_{*}\right)$ and the adiabatic SW effect is given by approximately $1 / 3 \Psi\left(\eta_{*}\right)$, the early ISW ridge is more prominent than the SW ridge in adiabatic models. However for scales that enter the horizon during matter domination, the decay in the potential due to radiation is much less significant. Thus the height of the second ridge drops significantly at larger scales (see Fig. 4a).

After $\Lambda$ domination $a / a_{0} \gtrsim\left(\Omega_{0} / \Omega_{\Lambda}\right)^{1 / 3}$, the potential once again decays. Note however that for typical values of $\Omega_{0} \gtrsim 0.1$, this decay begins only comparatively recently leading to late ISW contributions. This has three significant consequences.

(a) The largest $k$-modes contribute little to the anisotropy due to the projection effect. Notice that the late ISW contribution intersects the $\ell=2$ edge of Fig. 4 at a smaller scale than the SW effect. In Fig. 5a, we plot the analytic decomposition of contributions to a $k$-mode slice corresponding to these large scales. The smaller late ISW contribution in fact partially cancels the SW effect. Since the SW contribution has not undergone free streaming oscillations at $\Lambda$ domination, the two effects contribute coherently and cancel due to the decay of the potential.

* For representation purposes, we chose the initial weights of the $k$-modes to correspond to $\left|C_{A}\right|^{2} \propto \tilde{k}$ and $\left|C_{I}\right|^{2} \propto \tilde{k}^{-3}$ for the adiabatic and isocurvature modes respectively. This does not sacrifice generality since one can easily scale the figure to an alternate initial weighting. Note all contour plots of the anisotropy represent the numerical results. 
(b) Since the potential is still decaying at the present, the late ISW effect can boost the low order multipoles for all scales. In Fig. 5b, we plot a smaller mode and show that the late ISW effect is positive definite. Recent contributions have not free streamed to the oscillatory regime. The ridge structure of Fig. 4 is due to the late ISW effect adding with every other ridge in the SW free streaming oscillation.

(c) Contributions are spread out over a time comparable to $\eta_{0}$. As we shall see in $\S \mathrm{VB}$, this implies cancellation of the late ISW contribution as the photon travels through many wavelengths of the perturbation [27]. Thus late ISW contributions are rapidly damped as the scale decreases leaving only those scales that project onto large angles.

Together these factors imply that if the $k$-modes are equally weighted (scale invariant), the result will be a rise toward low multipoles from the late ISW contributions [28]. On the other hand if scales that are superhorizon sized at late ISW generation are strongly weighted, there is a relative suppression of low multipoles due to SW and ISW cancellation.

Open adiabatic models follow similar physical principles yet still yield significantly different anisotropies. Both the late ISW contribution at large scales and the early ISW contribution at small scales contributes near the maximum of $2 \Psi\left(\eta_{*}\right)$. On most scales, the combined ISW effect completely dominates over the SW contributions (see Fig. 6). However, just as in the $\Lambda$ case, the late ISW contributions boost the anisotropy in a larger angle than the SW effect for a given $k$-mode. Notice where the late ISW ridge intersects $\ell=2$. For the largest mode $k=\sqrt{-K}$, the SW effect consequently can contribute mildly and cancel part of the late ISW effect as in the $\Lambda$ case. Moreover, this projection effect implies that at these scales, the late ISW effect itself is increasingly suppressed with $\ell$ (see Fig. 5c). These curvature scale contributions however are suppressed by the cut off in the potential near the curvature scale from the Poisson equation (13). This has the effect of converting the SW ridge into a peaked structure and curves the contours of the ISW ridge away from $k=\sqrt{-K}$. Of course a change in the underlying power spectrum that weights the $k$-modes can partially or completely remove this effect.

At smaller scales, the late ISW effect completely dominates the low order multipoles (see Fig. 5d). Finally notice the evolutionary effect of geodesic deviation. Comparing Figs. 4 and 6 , we see that the fluctuations are more rapidly carried to high multipoles than in the $\Lambda$ case.

Isocurvature models differ significantly in that the potentials grow until full matter domination. Strong early ISW contributions which are qualitatively similar to the SW term will occur directly after recombination and continue until full matter domination (see Fig. 2). Thus the projection of scales onto angles will follow a continuous sequence which merges the SW and early ISW ridges (see Fig. 7).

For the $\Lambda$ case, the early ISW effect completely dominates that of the late ISW effect. Thus the analytic separation shows that the total ISW and SW effects make morphologically similar contributions and the boost in low order multipoles is not manifest. Moreover, the two add coherently creating a greater total effect unlike the adiabatic case (see Fig. 5a,b). Open isocurvature models behave similarly except that the late ISW contributions near its maximum (late ISW ridge) is not negligible. It is thus similar to the adiabatic case (c.f. Fig. 5d and 8d) except that it does not usually dominate the total anisotropy.

Notice also that since there is no curvature cutoff in the potentials, the SW and early ISW ridge extends all the way to the largest mode $k=\sqrt{-K}$. The projection ridge intersects $k=\sqrt{-K}$ at $\ell \simeq 10$ which is the scale the $\left(\Omega_{0}=0.1\right)$ curvature radius subtends at a distance $\eta_{0}$ (see Fig. 8a and Appendix A). This 
is indicative of the fact that the lowest eigenmodes $k \rightarrow \sqrt{-K}, \tilde{k} \rightarrow 0$ all contribute to curvature scale fluctuations (see §VIB and Appendix A).

\section{Intermediate Scale Evolution: Acoustic Oscillations and Standard Recombination}

As the perturbation enters the horizon, we can no longer view the system as a single fluid. Decoupled components such as the neutrinos free stream and change the entropy fluctuation. However, above the photon diffusion scale, the photons and baryons are still tightly coupled until last scattering. Since even at recombination, the diffusion length is much smaller than the horizon $\eta_{*}$, it is appropriate to combine the photon and baryon fluids for study $[30,31,32]$. In this section, we show that photon pressure resists the gravitational compression of the photon-baryon fluid, leading to driven acoustic oscillations [6]

\section{A. The Acoustic or "Doppler" Peaks}

At intermediate scales, neither radiation pressure nor gravity can be ignored. Fortunately, their effects can be analytically separated and analyzed. Since photon-baryon tight coupling still holds, it is appropriate to expand the Boltzmann equation (6) and the Euler equation (14) for the baryons in the Compton scattering time $\dot{\tau}^{-1}$ [33]. To zeroth order, we obtain $\Theta_{1} \equiv V_{\gamma}=V_{b}$, and $\Theta_{\ell}=0$ for $\ell \geq 2$. There is therefore no Doppler shift from a scattering event, since the photons are already isotropic in the baryon rest frame. Again this implies that the photon-baryon evolution is adiabatic $\dot{\Delta}_{b}=3 / 4 \dot{\Delta}_{\gamma}$ even when the general evolution is not. The first order expansion yields

$$
\ddot{\Theta}_{0}+\frac{\dot{R}}{1+R} \dot{\Theta}_{0}+k^{2} c_{s}^{2} \Theta_{0}=F
$$

where the photon-baryon sound speed is

$$
c_{s}^{2}=\frac{1}{3} \frac{1}{1+R}
$$

and the forcing function is

$$
F=-\ddot{\Phi}-\frac{\dot{R}}{1+R} \dot{\Phi}-\frac{k^{2}}{3} \Psi
$$

The techniques established in our recent analysis of adiabatic fluctuations in a flat universe [6] work equally well for the general case. Under this formalism, the gravitational driving forces are treated as known external potentials in which the photon-baryon fluid oscillates. The right hand side determines the effect of gravity through the ISW effect and gravitational infall $k^{2} \Psi$. Notice that the SW effect due to the photon's subsequent climb out of the potential well partially counters infall [6]. The ISW term $\ddot{\Phi}$ also drives the oscillation and is important at horizon crossing for modes that cross during radiation domination. We shall see in $\S I V C$ that ISW contributions after last scattering can also counter or even overwhelm the SW term.

The left hand side of equation (32) represents an oscillator whose restoring force is due to the photon pressure. This homogeneous $F=0$ equation can be solved by the WKB approximation,

$$
\begin{aligned}
& \theta_{a}=(1+R)^{-1 / 4} \cos k r_{s}, \\
& \theta_{b}=(1+R)^{-1 / 4} \sin k r_{s},
\end{aligned}
$$

where the sound horizon is

$$
r_{s}=\int_{0}^{\eta} c_{s} d \eta^{\prime}=\frac{2}{3} \frac{1}{k_{e q}} \sqrt{\frac{6}{R_{e q}}} \ln \frac{\sqrt{1+R}+\sqrt{R+R_{e q}}}{1+\sqrt{R_{e q}}}
$$


with $R_{e q} \equiv R\left(\eta_{e q}\right)$. Notice that if the sound speed is constant, the dispersion relation becomes $\omega=k c_{s}$ as expected of acoustic oscillations. The solution in the presence of the source $F$, constructed by Green's method, is [6]

$$
\begin{array}{r}
{[1+R(\eta)]^{1 / 4} \Theta_{0}(\eta)=\Theta_{0}(0) \cos k r_{s}(\eta)+\frac{\sqrt{3}}{k}\left[\dot{\Theta}_{0}(0)+\frac{1}{4} \dot{R}(0) \Theta_{0}(0)\right] \sin k r_{s}(\eta)} \\
+\frac{\sqrt{3}}{k} \int_{0}^{\eta} d \eta^{\prime}\left[1+R\left(\eta^{\prime}\right)\right]^{3 / 4} \sin \left[k r_{s}(\eta)-k r_{s}\left(\eta^{\prime}\right)\right] F\left(\eta^{\prime}\right),
\end{array}
$$

and $k \Theta_{1}=-3\left(\dot{\Theta}_{0}+\dot{\Phi}\right)$. Although the potentials in $F$ can be approximated from their large (§III) and small (§IVB) scale solutions, to show the true power of this technique, we instead employ their numerical values in Fig. 9. The excellent agreement with the full solution indicates that our technique is limited only by our knowledge of the potentials. In almost all models, the potentials can at least be approximated from the calculated matter power spectrum at the present and the general principles of their evolution (see e.g. [6]).

Some common features of these acoustic oscillations, valid for both isocurvature and adiabatic fluctuations are worthwhile to note. At the start of the oscillation, the amplitude of the monopole increases with $R$ (i.e. $\Omega_{b} h^{2}$ ) due to a reduction in the pressure restoring force. Although both the monopole and the dipole subsequently decrease, the dipole does so more rapidly due to an additional factor of $\dot{r}_{s}=c_{s} \propto(1+R)^{-1 / 2}$. Thus, when last scattering freezes in the adiabatic oscillations, the temperature fluctuations will be dominated by the monopole. Furthermore, the amplitude of the monopole is itself modulated since inside a potential well, the compressional phase of the oscillation is enhanced and the expansion phase suppressed if $\Psi$ is comparable to $\Theta_{0}$ (see Fig. 9b). This can also be viewed as a shift in the zero point of the oscillations due to gravity. All even peaks for the adiabatic and odd peaks for the isocurvature models suffer this suppression. In models were the pressure is relatively low (high $\Omega_{b} h^{2}$ ), the expansion phase may be hidden entirely in the final anisotropy spectrum [6].

Those features which distinguish isocurvature from adiabatic fluctuations are also apparent by inspection. For adiabatic initial conditions, the driving potentials are constant until Jeans crossing, at which point they decay (see Fig. 9). On the other hand, for the isocurvature scenario, they grow from zero to a maximum at Jeans crossing. Thus the forcing function imitates $\cos k r_{s}$ and $\sin k r_{s}$ in the adiabatic and isocurvature scenario respectively and stimulates the corresponding mode of temperature fluctuations.

\section{B. From the Jeans to Diffusion Scale}

Well below the Jeans scale, the gravitational driving force can be ignored and the photon-baryon fluctuations behave as simple oscillatory functions, until the breakdown of tight coupling at the photon diffusion scale. At this point, photon fluctuations are exponentially damped due to diffusive mixing and rescattering. We can account for this by expanding the Boltzmann and Euler equations for the photons and baryons respectively to second order in $\dot{\tau}^{-1}$ [34]. This gives the dispersion relation an imaginary part, making the general solution

$$
\Theta_{0}=A(1+R)^{-1 / 4} \mathcal{D}(\eta, k) \cos k r_{s}+B(1+R)^{-1 / 4} \mathcal{D}(\eta, k) \sin k r_{s},
$$

where the damping factor is

$$
\mathcal{D}(\eta, k)=e^{-\left(k / k_{D}\right)^{2}},
$$


with the damping scale

$$
k_{D}^{-2}=\frac{1}{6} \int d \eta \frac{1}{\dot{\tau}} \frac{R^{2}+4(1+R) / 5}{(1+R)^{2}} .
$$

Diffusion thus dissipates these acoustic waves leading to energy input and spectral distortions in the CMB $[35,36]$.

The amplitudes of these oscillations, i.e. the constants $A$ and $B$, are determined by the total effect of the gravitational driving force in equation (32). However, a simpler argument suffices for showing its general behavior. As shown in $\S$ IIIB, isocurvature fluctuations grow like $\Delta_{\gamma} \simeq-a C_{I}$ until Jeans crossing. Since the Jeans crossing time is $a_{J} \sim k_{e q} / k$, the isocurvature amplitude will be suppressed by $k_{e q} / k$. On the other hand, adiabatic fluctuations which grow as $a^{2}$ will have a $\left(k_{e q} / k\right)^{2}$ suppression factor. This simple argument fixes the amplitude up to a factor of order unity.

We obtain the specific amplitude by solving equation (16) under the constant entropy assumption $\dot{S}=0$. The latter approximation is not strictly valid since free streaming of the neutrinos will change the entropy fluctuation. However, since the amplitude is fixed after Jeans crossing, which is only slightly after horizon crossing, it suffices. Under this assumption, the equation can again be solved in the small scale limit. Kodama \& Sasaki [8] find that for isocurvature fluctuations,

$$
A=0, \quad B=-\frac{\sqrt{6}}{4} \frac{k_{e q}}{k} C_{I}, \quad \text { (iso) }
$$

whereas for adiabatic perturbations,

$$
A=\frac{5}{4}\left(\frac{k_{e q}}{k}\right)^{2} C_{A}, \quad B=0, \quad(\text { adi })
$$

if $k \gg k_{e q}$ and $k \eta \gg 1$. As expected, the isocurvature mode stimulates the $\sin k r_{s}$ harmonic, as opposed to $\cos k r_{s}$ for the adiabatic mode.

We can also construct the evolution of density perturbations at small scales. Well inside the horizon, $\Delta_{\gamma}=4 \Theta_{0}$ by equation (11). The isocurvature mode solution therefore satisfies (RD/MD)

$$
\Delta_{\gamma} / C_{I}=-\sqrt{6}\left(\frac{k_{e q}}{k}\right)(1+R)^{-1 / 4} \mathcal{D}(a, k) \sin k r_{s} .
$$

The tight coupling limit implies $\dot{\Delta}_{b}=3 / 4 \dot{\Delta}_{\gamma}$ which requires (RD/MD),

$$
\Delta_{b} / C_{I}=1-\frac{3 \sqrt{6}}{4}\left(\frac{k_{e q}}{k}\right)(1+R)^{-1 / 4} \mathcal{D}(a, k) \sin k r_{s} .
$$

This diffusive suppression of the adiabatic component for the baryon fluctuation is known as Silk damping [12]. After damping, the baryons are left with the original entropy perturbation $C_{I}$. Since they are surrounded by a homogeneous and isotropic sea of photons, the baryons are unaffected by further photon diffusion. From the photon or baryon continuity equations at small scales, we obtain (RD/MD)

$$
V_{b} / C_{I}=V_{\gamma} / C_{I} \simeq \frac{3 \sqrt{2}}{4}\left(\frac{k_{e q}}{k}\right)(1+R)^{-3 / 4} \mathcal{D}(a, k) \cos k r_{s}
$$


As one would expect, the velocity oscillates $\pi / 2$ out of phase with, and increasingly suppressed compared to, the density perturbations. Employing equations (43) and (44), we construct the total density perturbation by assuming that free streaming has damped out the neutrino contribution ( $\mathrm{RD} / \mathrm{MD})$,

$$
\Delta_{T} / C_{I}=\frac{a}{1+a}\left[1-\frac{3 \sqrt{6}}{4} \frac{k_{e q}}{k} R^{-1}(1+R)^{3 / 4} \mathcal{D}(a, k) \sin k r_{s}\right],
$$

From this equation, we may derive the potential (RD/MD),

$$
\Psi / C_{I}=-\frac{3}{4}\left(\frac{k_{e q}}{k}\right)^{2} \frac{1}{a}\left[1-\frac{3 \sqrt{6}}{4} \frac{k_{e q}}{k} R^{-1}(1+R)^{3 / 4} \mathcal{D}(a, k) \sin k r_{s}\right]
$$

which decays with the expansion since $\Delta_{T}$ goes to a constant. In Fig. 10, we compare these analytic approximations with the numerical results. After damping eliminates the adiabatic oscillations, the evolution of perturbations is governed by diffusive processes.

A similar analysis for adiabatic perturbations shows that diffusion damping completely eliminates small scale baryonic fluctuations. Unlike the isocurvature case, unless CDM wells are present to reseed fluctuations (see $\S \mathrm{V})$, adiabatic models consequently fail to form galaxies. All adiabatic examples shown here, including the open ones, are for CDM universes.

\section{Recombination and Free Streaming}

If last scattering occurs before diffusion has damped the acoustic oscillations in a given mode, e.g. in the standard recombination models, they will be frozen into the CMB. A generalization of the free streaming equation of (31) gives the resulting anisotropies,

$$
\begin{aligned}
& \frac{\Theta_{\ell}(\eta)}{2 \ell+1}=\left[\Theta_{0}+\Psi\right]\left(\eta_{*}\right) X_{\nu}^{\ell}\left(\chi-\chi_{*}\right)+\Theta_{1}\left(\eta_{*}\right) \frac{1}{k} \frac{d}{d \eta} X_{\nu}^{\ell}\left(\chi-\chi_{*}\right) \\
&+\int_{\eta_{*}}^{\eta}(\dot{\Psi}-\dot{\Phi}) X_{\nu}^{\ell}\left(\chi-\chi^{\prime}\right) d \eta^{\prime} .
\end{aligned}
$$

Here we obtain the diffusion damped fluctuation at last scattering from equation (37) by the replacements $[6]$

$$
\begin{aligned}
{\left[\Theta_{0}+\Psi\right]\left(\eta_{*}\right) } & \rightarrow\left[\Theta_{0}+\Psi\right]\left(\eta_{*}\right) \mathcal{D}\left(\eta_{*}, k\right), \\
\Theta_{1}\left(\eta_{*}\right) & \rightarrow \Theta_{1}\left(\eta_{*}\right) \mathcal{D}\left(\eta_{*}, k\right),
\end{aligned}
$$

where the damping factor is averaged over the finite duration of last scattering

$$
\mathcal{D}\left(\eta_{*}, k\right)=\int d \eta \dot{\tau} e^{-\tau} e^{-\left(k / k_{D}\right)^{2}} .
$$

Since the visibility function $\dot{\tau} e^{-\tau}$ goes to a delta function for large $\tau$, this definition also coincides with the tight coupling limit, equation (39). For analytic approximations of the visibility function see [6,37].

As we have shown in [6], equation (48) describes the final anisotropies due to acoustic oscillations to high accuracy for any given model. However, for the task of reconstructing the model from observations, it is useful to have a simple estimate of equation (48). As we have already seen with the Sachs-Wolfe effect, the presence of the radial eigenfunctions $X_{\nu}^{\ell}$ in equation (48) merely represents the projection of a spatial scale onto an angular scale on the sky today. The wavenumbers of the peaks in the spectrum 
will correspond to the modes in which the monopole reaches an extrema at last scattering: for adiabatic fluctuations $k_{m}=m \pi / r_{s}\left(\eta_{*}\right)$, whereas for isocurvature fluctuations $k_{m}=(m-1 / 2) \pi / r_{s}\left(\eta_{*}\right)$, where $m$ is an integer $\geq 1$. The first oscillation is thus at approximately the sound horizon at last scattering $r_{s}\left(\eta_{*}\right)$. This fluctuation is seen as an anisotropy in the multipole $\ell_{m} \simeq k_{m} r_{\theta}$, which corresponds to the angle subtended by the scale $k_{m}$ at the distance of the last scattering surface. In the small angle approximation

$$
r_{\theta}=\frac{1}{\sqrt{-K}} \sinh \left[\chi_{0}-\chi_{*}\right]
$$

which reduces to $r_{\theta}=\eta_{0}-\eta_{*}$ for a flat universe.

The rapid deviation of geodesics in an open universe causes a given scale to subtend a smaller angle on the sky. In older universes (large present horizon $\eta_{0}$ from $h$ or $\Lambda$ ), the distance to the last scattering surface increases, also reducing the angle a given scale subtends. However, this effect tends to cancel with the corresponding increase in the sound horizon at last scattering. The imperfect cancellation in the $\Lambda$ case pushes the peak to somewhat smaller angles.

In Fig. 11, we plot the angular location $\ell_{p}=\pi r_{\theta} / r_{s}\left(\eta_{*}\right)$ from which the series of peaks can be obtained as described above. This simple estimate does remarkably well in tracing the higher peaks which are dominated by pure acoustic effects. For the first peak, the potentials are still large enough at last scattering to play a subsequent role. As mentioned in §IVA, the potential decays after horizon crossing due to radiation growth suppression if the universe is not fully matter dominated. For scales that cross the horizon before last scattering, this drives the acoustic oscillations. Afterwards it changes the gravitational redshift that the photon would otherwise suffer from the SW effect. We call the latter the early ISW effect. Since the sound horizon is always smaller than the particle horizon, it contributes to scales between the first acoustic peak and scales which cross the horizon during full matter domination.

For the adiabatic mode, the early ISW effect partially or fully removes the SW redshift, uncovering the intrinsic fluctuation $\Theta_{0}\left(\eta_{*}\right)$ from the initial conditions and adiabatic growth, as well as a contribution up to $\Phi\left(\eta_{*}\right)$ from the time dilation. This can be seen in the additional contributions to the rms temperature fluctuations after the fluctuation crosses the horizon in Fig. 12a. Since the potential has already decayed by curvature or $\Lambda$ domination, further decay does not significantly affect the radiation. For scales that cross well after radiation domination, the late ISW effect is more significant and serves to distinguish between $\Lambda$ and open models. At intermediate scales, both effects are important. However, since the late ISW contributions are cancelled at small scales (see $\S \mathrm{VB}$ ), the total anisotropy is relatively smaller here.

For isocurvature models, the potential continues to grow outside the horizon in the radiation dominated epoch (see Fig. 12b). Therefore, evolution weights the large scales more heavily than the small. In fact, the first acoustic oscillation at last scattering is not prominent due both to this enhancement of the large scale and the continuing growth of the potential which enhances the second oscillation. This evolutionary tilt toward large scale anisotropies can be countered by changing the initial power spectrum. However, the corresponding enhancement of small scale matter fluctuations makes reionization likely in this model. In this case, anisotropies are destroyed and then regenerated by diffusive effects. It is to this subject, we now turn. 


\section{Small Scale Evolution: Diffusion Effects and Reionization}

Below the photon diffusion length, even photon-baryon tight coupling breaks down. Since the photons diffuse amongst the baryons, the two fluids must be treated separately. Moreover, if the universe is reionized, the diffusion length can grow to nearly the horizon size at last scattering. The window for adiabatic oscillations closes, and degree scale anisotropies in the CMB will be dominated by diffusive effects. Isocurvature baryon models also behave quite differently from adiabatic CDM models with respect to the matter. For the decoupled CDM, density perturbations grow regardless of ionization, providing potential wells into which the baryons may later fall. Their absence in the baryonic isocurvature case makes the ionization history a crucial ingredient for structure formation under this scenario. Consequently although we retain generality for $\mathrm{CMB}$ anisotropies, we concentrate on the isocurvature model when discussing the effects of reionization on the matter.

\section{A. Matter Evolution: Compton Drag}

\section{Partial or Full Ionization}

Due to the lack of Silk damping, baryon isocurvature models typically have high amplitude small scale fluctuations which can collapse immediately after standard recombination at $z \simeq 1000$ [2]. It is possible that enough energy is released to immediately reionize some fraction $x_{e}$ of the electrons. This model will effectively behave as if recombination did not occur at all.

Although the tight coupling approximation predicts $V_{b}$ and $\Delta_{\gamma}$ go to zero inside the diffusion length, its breakdown keeps this from being exactly satisfied (c.f. Fig. 10). The single fluid Jeans argument of $\S I I I$ become invalid. Since in the diffusion limit where $S \simeq \Delta_{b} \gg \Delta_{\gamma}$, the effect of radiation pressure on $\Delta_{T}$ in equation (16) is exactly canceled by the entropy term. After complete matter domination, the evolution equation and its solution therefore becomes identical to the pressureless case, i.e. all modes grow by the same factor $D(a)$ [see equation (19)].

We can quantify this with the Compton drag argument of $\S$ IIC. After $z_{d}=160\left(\Omega_{0} h^{2}\right)^{-1 / 5} x_{e}^{-2 / 5}$, the baryons are effectively released from photon pressure. Thus, perturbations will grow such that $\Delta_{T}(a)=$ $\left[D(a) / D\left(a_{d}\right)\right] C_{I}$ for $a \gg a_{d}$. An excellent empirical approximation (see Fig. 13) to the behavior at intermediate times is given by

$$
\Delta_{b} / C_{I}=\mathcal{G}\left(a, a_{d}\right)
$$

with the interpolation function

$$
\mathcal{G}\left(a_{1}, a_{2}\right)=1+\frac{D\left(a_{1}\right)}{D\left(a_{2}\right)} \exp \left(-a_{2} / a_{1}\right)
$$

where if $a_{1} \gg a_{2}, \mathcal{G}\left(a_{1}, a_{2}\right) \rightarrow D\left(a_{1}\right) / D\left(a_{2}\right)$. The velocity $V_{T}$ is given by the continuity equation (16). Notice that this properly accounts for growth in an open and/or $\Lambda$ universe. 


\section{Late Ionization}

Now let us consider more complicated thermal histories. Standard recombination may be followed by a significant transparent period before reionization at $z_{i}$, due to some later round of structure formation. There are two effects to consider here: fluctuation behavior in the transparent regime and after reionization. Let us begin with the first question. Closely following recombination, the baryons are released from drag essentially at rest and thereafter can grow in pressureless linear theory. The joining conditions then imply that $3 / 5$ of the perturbation joins the growing mode [34], yielding present fluctuations of $\sim 3 /{ }_{5} C_{I} D(z=0) / D(z \sim 800)$ where the residual ionization makes the drag epoch $z \sim 800<z_{*}$. The evolution is again well described by the interpolation function (52) so that $\Delta_{b}(a)=\mathcal{G}\left(a, a_{t}\right) C_{I}$. By this argument, the effective redshift to employ is $z_{t} \sim 3 / 5800 \simeq 400-500$. We take here $z_{t} \simeq 450$.

Now let us consider the effects of reionization at $z_{i}$. After $z_{i}$, Compton drag again prevents the baryon perturbations from growing. Therefore the final perturbations will be $\Delta_{b}\left(a_{0}\right) \simeq \Delta_{b}\left(a_{i}\right) D\left(a_{0}\right) / D\left(a_{d}\right)$. Joining the transparent and ionized solutions, we obtain

$$
\Delta_{b} / C_{I}= \begin{cases}\mathcal{G}\left(a, a_{t}\right) & a<a_{g} \\ \mathcal{G}\left(a_{i}, a_{t}\right) \mathcal{G}\left(a, a_{d}\right), & a>a_{g}\end{cases}
$$

which is plotted in Fig. 14. Since perturbations do not stop growing immediately after reionization and ionization after the drag epoch does not affect the perturbations, we take $a_{g}=\min \left(1.1 a_{i}, a_{d}\right)$.

\section{B. Photon Evolution: The Doppler and Small Scale Effects}

We now need to examine the evolution of photon temperature perturbations in light of these results for the matter. As the diffusion length overtakes the fluctuation, acoustic oscillations in the photons are washed away (see Fig. 15). Since the baryon velocity can grow after $z_{d}$ so that $V_{b} \gg V_{\gamma}$, Doppler shifts off moving electrons will regenerate temperature perturbations. Yet since $k>k_{D}$, these perturbations will be erased as the photons travel across several wavelengths of the perturbation and are rescattered. Unlike the acoustic oscillations, photon evolution before last scattering is inconsequential. This is true even in late ionization scenarios. The large acoustic fluctuations frozen in at recombination become anisotropies as they free stream to the reionization epoch where they are damped as $e^{-\tau}$ by rescattering. Thereafter, fluctuations are regenerated by the Doppler effect at last scattering exactly as in the partially ionized case. Doppler anisotropies therefore can be completely described by the matter fluctuations at last scattering [38].

Moreover, since the optical depth decreases only due to the expansion, last scattering will extend for a period of time comparable to $\eta_{*}$. The later last scattering is, the thicker the last scattering surface. Cancellation between photons which last scattered off a crest or trough of the matter perturbation will severely damp the Doppler effect on scales smaller than the thickness. Together, this implies that the higher the ionization fraction $x_{e}$, the more severely damped these anisotropies will be (see Fig. 15). One must be careful however to avoid overproducing spectral distortions in the CMB due to scattering off hot reionized electrons. Under most plausible ionization scenarios, fully ionized open models are ruled out by the low Compton- $y$ distortion [4].

We can analytically account for these effects by using the weak coupling approximation [38] which treats the photons as diffusing across independently evolving baryon perturbations. Moreover, due to the cancellation of the Doppler effect, ordinarily negligible contributions become significant and must also be included 
in this formalism, e.g. the late ISW effect [27] and second order contributions [3,39]. The dominant second order correction, called the Vishniac effect $[40,41]$ couples $V_{b}$ to the spatial dependence of the scattering probability, i.e. since $n_{e} \simeq\left\langle n_{e}\right\rangle\left(1+\Delta_{b}\right)$, let $\dot{\tau} \rightarrow \dot{\tau}\left(1+\Delta_{b}\right)$ in equation (3). Note that this effect is not present in the numerical calculation. Ignoring curvature and taking the ordinary Fourier transform, we obtain the formal solution for the $k$ th mode of the Boltzmann equation [27],*

$$
[\Theta+\Psi](\eta, k, \mu)=[\Theta+\Psi]\left(\eta_{d}, k, \mu\right) e^{i k \mu\left(\eta_{d}-\eta\right)} e^{-\tau\left(\eta_{d}, \eta\right)}+\left[\Theta_{D S W}+\Theta_{I S W}+\Theta_{V}\right](\eta, k, \mu),
$$

with $k \mu=\mathbf{k} \cdot \gamma$, the optical depth $\tau\left(\eta_{1}, \eta_{2}\right)=\int_{\eta_{1}}^{\eta_{2}} \dot{\tau} d \eta, \Theta_{D S W}$ the Doppler and SW contributions, $\Theta_{I S W}$ the late ISW effect and $\Theta_{V}$ the Vishniac effect. As noted above, scattering rapidly damps out the contributions from before the drag epoch as $e^{-\tau}$. Thus the photon temperature perturbation is a function of the matter perturbations alone. These source terms are explicitly given by

$$
\begin{aligned}
\Theta_{D S W}(\eta, k, \mu) & =\int_{\eta_{d}}^{\eta}\left(\Theta_{0}+\Psi-i \mu V_{b}\right) \dot{\tau} e^{-\tau\left(\eta^{\prime}, \eta\right)} e^{i k \mu\left(\eta^{\prime}-\eta\right)} d \eta^{\prime}, \\
\Theta_{I S W}(\eta, k, \mu) & =\int_{\eta_{d}}^{\eta} 2 \dot{\Psi} e^{-\tau\left(\eta^{\prime}, \eta\right)} e^{i k \mu\left(\eta^{\prime}-\eta\right)} d \eta^{\prime}, \\
\Theta_{V}(\eta, k, \mu) & =\int_{\eta_{d}}^{\eta}-i \sum_{k^{\prime}} \mu^{\prime} V_{b}\left(\eta^{\prime}, k^{\prime}\right) \Delta_{b}\left(\left|\mathbf{k}-\mathbf{k}^{\prime}\right|\right) \dot{\tau} e^{-\tau\left(\eta^{\prime}, \eta\right)} e^{i k \mu\left(\eta^{\prime}-\eta\right)} d \eta^{\prime},
\end{aligned}
$$

where recall that the plane wave decomposition is defined such that $\gamma \cdot \mathbf{v}_{b}(\eta, \mathbf{x})=-i \mu V_{b}(\eta, k) \exp (i \mathbf{k} \cdot \mathbf{x})$ [see equation (5)]. The visibility function $\dot{\tau} e^{-\tau}$ picks out the epoch of last scattering, and the second order nature of the Vishniac term is reflected in the mode coupling sum.

For scales smaller than the thickness of the last scattering surface, as determined by the width of the visibility function $\dot{\tau} e^{-\tau}$, the Doppler, SW, and Vishniac effects, will be cancelled by oscillation in the integrand of equation (55) for all but the perpendicular $\mu=0$ mode. Analogously $\dot{\Psi} e^{-\tau}$ defines a thickness of the "gravitational last scattering surface" under which contributions are also cancelled.

Linear theory flows are irrotational, $\gamma \cdot \mathbf{v} \propto \mu k$, and gravitational redshifts are absent in the direction perpendicular to the oscillation. Both the Doppler effect and the SW effect thus vanish for $\mu=0$, implying severe cancellation. Because cancellation occurs similarly and $|\Psi| \ll\left|V_{b}\right|$ on small scales, the residual SachsWolfe effect will be much smaller that the other two effects. By angularly averaging the first of equations (54), we obtain the residual effect on the monopole [38],

$$
\Theta_{0} \simeq \frac{1}{4} \Delta_{\gamma} \simeq \frac{V_{b}}{k} \dot{\tau}
$$

which feeds back through equation (54) into the uncanceled $\mu=0$ mode [41]. This $\mu=0$ mode is also how all effects avoid cancellation. Small scale density perturbations, with oscillations perpendicular to the line of sight, can be in bulk motion parallel to the line of sight. The result is the Vishniac effect: a small scale temperature anisotropy due to the increase in probability of scattering off an overdense region. The late ISW effect is similar. But note that in the $\Lambda$ case, the thickness is comparable to $\eta_{0}$ implying cancellation

* Since the Vishniac effect is not linear, we must consider $k$-mode coupling. Therefore, in this and the following sections where power spectra are employed, we restore the $k$-index of the perturbations to avoid confusion. 
occurs up to scales near the present horizon. Thus whereas in the open case one sees a gentle roll off of contributions in $\ell$, in the $\Lambda$ case, anisotropies fall sharply even from the lowest $\ell$.

This method is valid for calculating these secondary fluctuations for either the isocurvature or adiabatic scenario, under any ionization history in which last scattering occurs after the end of the drag epoch.* In Fig. 10, we show that this approximation (56) compares well with the numerical result roughly between the drag epoch and last scattering, as expected.

Integration of equation (55) determines the present rms temperature perturbations as a function of the underlying matter fluctuations $P(k)=\left|\Delta_{T}\left(\eta_{0}, k\right)\right|^{2}$. For late last scattering, the integrands in equation (55) are wide bell shaped functions. The functions $\Theta_{D S W}$ and $\Theta_{I S W}$ are therefore approximately Fourier transforms whose contribution to the rms can be approximated using Parseval's theorem [39, 41, 38]

$$
|\Theta+\Psi|_{r m s}^{2}\left(\eta_{0}, k\right)=\pi \frac{P(k)}{\left(k \eta_{0}\right)^{5}} \int_{0}^{1} d x\left[\left|G_{D S W}(x)+G_{I S W}(x)\right|^{2}+\left|G_{V}(x)\right|^{2} I_{V}(k)\right],
$$

where $x=\eta / \eta_{0}$. The growth is accounted for by the conformal time integrals over

$$
\begin{aligned}
G_{D S W}(x) & =\eta_{0}^{3}\left[\frac{\ddot{D}}{D_{0}} \dot{\tau}+\frac{\dot{D}}{D_{0}} \ddot{\tau}\right] e^{-\tau\left(\eta, \eta_{0}\right)} \\
G_{I S W}(x) & =3\left(\frac{a_{0}}{a}\right)^{2} \eta_{0}^{3} H_{0}^{2} \Omega_{0}\left[\frac{\dot{D}}{D_{0}} \frac{a}{a_{0}}-\frac{D}{D_{0}} \frac{\dot{a}}{a_{0}}\right] e^{-\tau\left(\eta, \eta_{0}\right)}, \\
G_{V}(x) & =\frac{\dot{D}}{D_{0}} \frac{D}{D_{0}} \dot{\tau} \eta_{0}^{2} e^{-\tau\left(\eta, \eta_{0}\right)}
\end{aligned}
$$

where $D_{0}=D\left(\eta_{0}\right)$ and the time independent mode coupling for the Vishniac effect is [39]

$$
I_{V}(k)=\frac{V}{16 \pi^{2}} \frac{\left(k \eta_{0}\right)^{5}}{\eta_{0}^{3}} \int_{0}^{\infty} d y \int_{-1}^{1} d(\cos \theta) \frac{\left(1-\cos ^{2} \theta\right)(1-2 y \cos \theta)^{2}}{\left(1+y^{2}-2 y \cos \theta\right)^{2}} \frac{P\left[k\left(1+y^{2}-2 y \cos \theta\right)^{1 / 2}\right]}{P(k)} \frac{P(k y)}{P(k)} .
$$

The Vishniac effect peaks strongly to small scales, whereas the first order Doppler and integrated Sachs-Wolfe contribution have the same scale dependence reflecting the cancellation process [27].

In summary, cancellation occurs because in the diffusive and free streaming limit photons travel through many wavelengths of the matter fluctuation source. Cancellation is particularly severe for the Doppler and SW effects due to a lack of a perpendicular mode, but is also present for the late ISW and Vishniac effects.

* For baryonic compact object dominated models, the density of free electrons may be so depleted that last scattering occurs before the drag epoch even with maximal ionization. Though the analysis is more complicated, it remains true that scattering attempts to equalize $V_{b}$ and $V_{\gamma}$. This boosts $V_{b}$ and suppresses $V_{\gamma}$ at large scales and vice versa at small scales [4]. 


\section{Matter \& Temperature Power Spectra}

The relative amplitudes of the $k$-modes which form the power spectrum are often set by an $a b$ initio anzatz. Taking a more agnostic approach which allows for future empirical determination of the weights, we examine the transfer functions, e.g. $P(k) \equiv\left|\Delta_{T}\left(a_{0}, k\right)\right|^{2}=\left|T(k) C_{I}(k)\right|^{2}$ and $\left|T(k) C_{A}(k)\right|^{2}$ for isocurvature and adiabatic matter perturbations respectively. For CMB anisotropies, each $\ell$-mode evolves differently and thus possesses its own transfer function. We present here the full $\ell-k$ space structure of the anisotropy transfer function. To illustrate the effects of altering the $k$-weighting, we also present a few specific examples for the underlying spectrum. Perhaps the simplest possible choice is a random phase pure power law in $\tilde{k}$ initially, i.e. $\left|C_{A}\right|^{2} \propto \tilde{k}^{n}$ and $\left|C_{I}\right|^{2} \propto \tilde{k}^{n}$ for adiabatic and isocurvature modes respectively. Although this may not be realistic near the curvature scale where geometric effects can introduce novel features [42], these toy models do illuminate the general case.

\section{A. Matter Transfer Function}

\section{Adiabatic Models}

For adiabatic models, the matter transfer function is affected by the dynamics and matter content only. Since in low $\Omega_{0}$ models, matter-radiation equality occurs late, the scale at which the transfer function turns over due to radiation growth suppression is larger. Furthermore, growth in the matter dominated epoch is suppressed due to curvature and/or $\Lambda$. Combining the standard fitting formula for the numerical results [43] with our analysis of growth rates, we may write the total transfer function as

$$
T(k)=D\left(\eta_{0}\right) \frac{\ln (1+2.34 q)}{2.34 q}\left[1+3.89 q+(14.1 q)^{2}+(5.46 q)^{3}+(6.71 q)^{4}\right]^{-1 / 4}
$$

where $q \equiv k /\left[\Omega_{0} h^{2} \exp \left(-2 \Omega_{b}\right)\right]$ and is valid for $\Omega_{b} \ll \Omega_{0}$. Aside from the small $\Omega_{b}$ dependence to account for coupling with the photons, $q \propto k / k_{e q}$. For scales that enter before equality, the perturbations grow as $a^{2}$ until Jeans crossing at $a_{J} \propto\left(k_{e q} / k\right)$. Thus the transfer function is flat at large scales and goes smoothly to $k^{-2}$ at small scales.

The definition of the adiabatic transfer funtion employed here carries information about the growth from equality to the present in the form of $D\left(\eta_{0}\right)$. However, in comparing different $\Omega_{0}$ models, the epoch of equality shifts. A more useful choice requires equal potentials $\Phi$ at the initial epoch (below the curvature scale). Specifically, this amounts to considering the quantity $T(k) /\left(\Omega_{0} h^{2}\right)^{2}$ due to the $k_{e q}^{2}$ from the Poisson equation (see Fig. 16).

This has the added benefit that the $k$-space SW contribution will also be the same. If the total ISW contributions after last scattering are negligible, this normalization of the transfer function is identical to a large angle anisotropy normalization for scale invariant spectra. For tilted spectra, one must account for changes in the $k$ to $\ell$ space projection through $\eta_{0}-\eta_{*}$. Since $n=1 \Omega_{\Lambda} \lesssim 0.9$ models approximately satisfy these conditions at the largest scales (see §IIID), the relative amplitude of anisotropy normalized matter fluctuations on various scales can be read directly from Fig. 16. Matter fluctuations at the $8 h^{-1} \mathrm{Mpc}$ $\left(k \simeq 0.1 \mathrm{Mpc}^{-1}\right)$ scale decrease in amplitude for fixed $h$ due to a change in equality rather than $\Lambda$ growth suppression [44].

For open adiabatic models, the situation is more complicated. As we have shown, the late ISW not the SW effect dominates the large angle anisotropies. From this we would expect that the anisotropy normalized 
matter amplitude would decrease relative to Fig. 16. However, this can be countered by the suppression of the gravitational potential (SW and ISW) effects from the curvature term in the Poisson equation (13). If the underlying spectrum is taken to be $\left|C_{A}\right|^{2} \propto \tilde{k}$, these effects in fact nearly cancel. However, we have reason to believe that the presence of a curvature scale may influence the initial conditions. For example, in the specific open inflationary case calculated in [42], the boost from the late ISW effect dominates and further suppresses matter fluctuations.

\section{Isocurvature Models}

In contrast to the adiabatic case, the isocurvature matter transfer function exhibits relatively complicated structure. On scales larger than the Jeans length, the matter gains a $k^{2}-3 K$ tail through the feedback mechanism (see §IIIB) and grow as $D(a)$ after radiation domination. Below this scale, the perturbations have damped oscillations around the initial conditions $C_{I}$ until the end of the drag epoch. Since the Jeans scale goes to a constant in the matter dominated epoch, this implies that the transfer function will have a significant peak at the maximal Jeans scale. Note that if the universe is not sufficiently matter dominated at last scattering, this scale could be less than its absolute maximum. Thus as the ionization fraction decreases, the peak in the transfer function moves to smaller scales (see Fig. 17). Since isocurvature models are motivated by the desire to satisfy observational estimates of $\Omega_{0} \simeq 0.2$, we will concentrate on the effects of ionization history rather than matter content.

We can in fact deduce some of the properties of the oscillatory regime from our simple analysis. In $\S \mathrm{IVB}$, we have shown that the oscillations decrease as $(1+R)^{-1 / 4} k_{e q} / k$ until they absolutely disappear for scales smaller than the diffusion length $k \gg k_{D}$, leaving a constant tail in the transfer function. However, after the drag epoch, all scales grow as $D(a)$ so that the flat tail will have an amplitude which is dependent on the ionization history. Notice furthermore that the oscillations become less prominent if last scattering is delayed, since both the $(1+R)^{-1 / 4}$ and diffusion suppression increases.

For $\Lambda$ models, the change in the growth rate boosts the amplitude of the transfer function. Since neither the Jeans scale nor the drag epoch depends on $\Lambda$ itself, the shape of the transfer function is the same aside from the lack of curvature effects at the largest scales. Analytic fitting formula may be adapted from the fully ionized case given by [45] modified to account for the growth rates presented in $\S$ VA.

Since large scale structure measurements indicate that $P(k) \propto k^{-1}$ at intermediate scales $10^{-2} \lesssim k / h \lesssim$ $1 \mathrm{Mpc}^{-1}$, which fall just below the maximal Jeans scale, the isocurvature scenario must have an initial spectrum of $n \simeq-1$ at least at these scales [46]. If the initial spectrum is assumed to be a single power law, this implies a very steep matter power spectrum at large scales since $\Delta_{T} \propto\left(k^{2}-3 K\right) C_{I}$. In other words, an isocurvature spectrum with index $n$ corresponds approximately to an adiabatic spectrum of $n+4$ at large scales, e.g. $n=-3$ yields scale invariance. The steep $n=-1$ implies large amounts of small scale power which allows for the early collapse of structure and early reionization [2]. 


\section{B. $C M B$ Anisotropies}

\section{Large Angles}

As we have shown in $\S$ IIID, the total Sachs-Wolfe effect can lead to interesting structure in the anisotropy at large scales in an $\Omega_{0}<1$ universe. But how dependent are the features on the underlying power spectrum? In Fig. 18, we show the full contributions to the anisotropy in both $\ell$ and $k$ as given by equation (9) for adiabatic models. Although we have chosen to represent a $\left|C_{A}\right|^{2} \propto \tilde{k}$ weighting, any initial power spectrum can be obtained from scaling by $\left|C_{A}\right|^{2} / \tilde{k}$. The full information of the two dimensional radiation transfer function is contained here. Notice that integration in $\log k$ yields the total anisotropy $\propto(2 \ell+1) C_{\ell}$ and in $\log \ell$ gives the rms temperature fluctuation for a given $k$-mode.

The adiabatic $\Omega_{0}=1$ case shown to full scale in the top left panel of Fig. 18, shows the tight $k-\ell$ correlation of the projection from last scattering (see §IIID). The SW effect contributes at large physical scales and the acoustic peaks at small physical scales. An expanded view in top right panel shows the break between the two effects around $\log \ell=1.5$ and $\log (k * \mathrm{Mpc})=-2$ for this model. Pivoting the underlying power spectrum around this value of $k$ simply emphasizes one effect over the other.

The situation is more complicated for $\Lambda$ and open models. The ISW term contributes to anisotropies for intermediate values of $k$. For low $\Omega_{0} \simeq 0.1-0.3$ the early ISW effect fills the gap between the SW ridge and acoustic peaks of the $\Omega_{0}=1$ model (see $\S$ IIID and IVC). The main contribution comes directly after horizon crossing for these intermediate $k$-values and thus projects onto lower $\ell$-modes than the SW effect.

At still larger scales, late ISW contributions become important. For $\Lambda$ models, they lead to low $\ell$ contributions since most fluctuations have not had time to free stream to high multipoles and those which have are cancelled. For intermediate $k$, the late ISW effect adds in quadrature to the SW effect. Yet for the largest $k$-modes, the SW effect itself has not free streamed and the late ISW effect will partially cancel it. Thus, depending on the $k$-weighting of the initial power spectrum, the late ISW term can have different effects. In Fig. 19, we plot the anisotropies for single power law weightings. Notice that the boost in the low multipoles from $\Lambda$ only occurs for intermediate values of the slope. On the other hand, for isocurvature models, the $\Lambda$ contributions to the late ISW effect are never prominent due to the dominance of the SW and early ISW effects.

For $\Omega_{0} \simeq 0.1-0.3$ open adiabatic universes, the total ISW effect almost always overwhelms the SW effect. There are two exceptions. Below a certain scale, the late ISW effect is thickness cancelled. Moreover these scales are often superhorizon sized at radiation domination so that the early ISW effect does not contribute either. At the largest scales, the projection carries the late ISW effect onto the unobservable monopole and dipole. Thus just as in the $\Lambda$ case, the relative weight of SW versus late ISW increases at large scales. Again, the SW and late ISW contributions at the largest scales tend to cancel. This is more important for curvature as opposed to $\Lambda$ late ISW contributions since the horizon size at curvature domination is smaller than that at $\Lambda$ domination. Indeed for somewhat higher $\Omega_{0}$ open models $\left(\Omega_{0} \simeq 0.5-0.8\right)$ where the SW and late ISW contributions are more comparable, cancellation can lead to a suppression of large angle anisotropies [5].

On the other hand, for the largest modes the amplitude of late ISW contribution itself decreases with $\ell$ due to the projection. Yet to have any net effect, the initial power spectrum must rise sharply to large scales to counter the $k^{2} / K$ Poisson equation suppression. Even the $k^{-1}$ rise toward large scales in recent predictions of an open inflationary model [42], does not overcome this suppression. Thus it is difficult to 
obtain a spectrum with falling anisotropies in open universe; in most cases the lowest order multipoles will show a rise in the anisotropy $\ell$ (see Fig. 19). This is often followed by a dip due to the transition between late and early ISW domination.

Open isocurvature models do not suffer Poisson suppression which makes curvature scale peculiarities manifest. Anisotropy contributions come from $k$ 's all the way to the curvature scale $k=\sqrt{-K}$ or $\tilde{k}=0$. Notice that this covers an infinite range in $\log \tilde{k}$, and yet the contributions retain exactly the same $\ell$-space structure. As we discuss in Appendix A, the radial eigenfunctions $X_{\nu}^{\ell}(\chi)$ have the peculiar property that even as the effective wavenumber $\nu=\tilde{k} / \sqrt{-K} \rightarrow 0$, they possess structure on order the curvature scale and are exponentially suppressed thereafter. Although the functions are complete, no random phase superposition of them will ever produce structure above the curvature scale. As $\tilde{k} \rightarrow 0$, all modes contribute at the angle the curvature scale subtends when the anisotropy was generated, e.g. at approximately the distance $\eta_{0}-\eta \simeq \eta_{0}$ for the SW and isocurvature early ISW effect.

For $\tilde{k}$-scale invariant potential, random phase weighting, the infinite number of decades in $\log \tilde{k}$ as $\tilde{k} \rightarrow 0$ causes a divergence in the anisotropy, if no cut off is assumed (see Fig. 20a). Moreover, any spectra that places even more power on scales $\tilde{k} \lesssim \sqrt{-K}$ will result in the same final anisotropy. This peculiarity can be seen in Fig. 19 for open isocurvature models with $n \lesssim-3$. Note however that " $\tilde{k}$-scale invariance" does not imply equal power on all physical scales since all low $\tilde{k}$ eigenfunctions have curvature scale power. Physical scales above the present horizon do not contribute to anisotropies despite the apparent divergence from low $\tilde{k}$. For the adiabatic case, the suppression of such scales from the Poisson equation prevents this effect from becoming manifest for reasonable $n$.

This indicates that for open isocurvature scenarios we must alter the power spectrum from $\tilde{k}$-scale invariance to have enough power at small scales to form galaxies. For spectra that are strongly tilted to small scales, anisotropies converge to approximately $\ell(2 \ell+1) C_{\ell} \propto \ell^{2}$ and become independent of $n$ and the model. This occurs for $n \gtrsim 1$ for isocurvature and $n \gtrsim 5$ for adiabatic conditions where recall that there is a $k^{4}$ difference in the correspondence of $n$ to the matter power spectrum. Because fluctuations are dominated by the smallest scale fluctuations present, i.e. those at the photon diffusion length $k_{D}$, equation (8) implies that $C_{\ell}$ is constant in $\ell$ as required. For an isocurvature scenario with index $-1 \lesssim n \lesssim 0$, which is of interest for structure formation, this asymptotic value has not yet been reached and $\ell(2 \ell+1) C_{\ell} \propto \ell$ approximately. This corresponds to an effective COBE DMR slope of $n_{\text {eff }} \simeq 2[5]$ implying that isocurvature models have significantly steeper anisotropy spectrum than the standard CDM model in which $n_{\text {eff }} \simeq 1$, but not as steep as one might naively think. In Fig. 20b, we show such an $n=-1$ weighting. Notice that bleeding from smaller $k$-modes than the main $k-\ell$ projection ridge is responsible for filling in the low $\ell$ anisotropy.

In summary, we have identified several independent causes of a downturn of anisotropies at low $\ell$ :

(a) The Poisson equation curvature cut off.

(b) SW and late ISW cancellation.

(c) Eigenfunction curvature cut off.

The first effect only occurs in open adiabatic models and manifests itself for $\Omega_{0} \lesssim 0.3$. The second effect is most significant when the SW and late ISW effects are comparable, e.g. open adiabatic models with $\Omega_{0} \simeq 0.5-0.8[5]$ and comes from scales which are superhorizon sized at the epoch of late ISW generation. The last effect applies if the initial spectrum gives significant weight to randomly phased low $\tilde{k}$ contributions 
and if the contributions are generated early enough to project onto an anisotropy instead of a monopole fluctuation, e.g. open isocurvature models with $n \lesssim 3$.

Two effects can give an upturn relative to the underlying power spectrum

(a) Late ISW contributions.

(b) High $k$-mode power bleeding into low $\ell$.

The late ISW effect predicts a rise toward low $\ell$ because of crest-trough cancellation at small scales. In a $\Lambda$ universe, this cutoff scale is on order the present horizon so that contributions are already falling sharply with $\ell$ at low $\ell$. For open universes, the late ISW effect contributes earlier and has a smaller scale cutoff. Thus the signature of the anisotropy transfer function is a rise to a plateau at low $\ell$. However, since the $k$-modes which contribute to this effect are the intermediate ones, this effect is only manifest if the initial spectrum gives them weight. For pure power laws, this requires a roughly scale invariant potential: $\tilde{k}^{3} \Phi^{2}=$ constant. In an open universe, the Poisson cutoff can change the plateau to a dip in the anisotropy at low $\ell$. For the opposite case of small scale weighted power spectra, $n \gtrsim-0.1$ for isocurvature and $n \gtrsim 3$ for adiabatic, the higher $k$-ridges in the projection effect contribute strongly to low $\ell$ multipoles. This implies that there is a maximum slope with which low order multipoles can rise, $C_{\ell} \simeq$ constant.

If any such features are detected in the observed spectrum and are statistically significant considering cosmic variance, some variation of the standard CDM picture will be necessary. However, even though a simple tilt (single power law) in the power spectrum cannot mimic such features, it is clear that more complicated initial spectra can. This degeneracy between the initial conditions and the evolutionary effects is lifted by assuming an ab initio model. In this case, large scale anisotropies are a simple yet powerful probe of the underlying cosmology as is well known. Alternatively, once the fundamental cosmological parameters, e.g. $\Omega_{0}, h, \Lambda$, are known, they will tell us what the initial conditions for structure formation are.

\section{Intermediate to Small Scale Anisotropies}

In standard recombination scenarios, acoustic oscillations determine the structure of anisotropies for both adiabatic and isocurvature modes. Since these oscillations contain a great deal of structure, it is obvious that more cosmological and model information can be extracted here than at larger angles. Moreover, once coverage of the sky at these angles becomes more complete, these measurements will be more immune to uncertainties from cosmic variance.

The angular scale of the peaks is determined by the projection of the sound horizon at last scattering onto the sky today and is independent of the underlying power spectrum. Three cosmological quantities, with their corresponding dependence on fundamental parameters, enter into its construction:

(a) $r_{s}\left(\eta_{*}\right) \simeq f\left(\Omega_{0} h^{2}, \Omega_{b} h^{2}\right)$, the sound horizon at last scattering,

(b) $\eta_{0}-\eta_{*}=f\left(\Omega_{0} h^{2}, h, \Omega_{\Lambda} h^{2}\right)$, the distance to the last scattering surface,

(c) $K=f\left(\Omega_{0} h^{2}, h, \Omega_{\Lambda} h^{2}\right)$, the curvature.

The first task is to distinguish between adiabatic and isocurvature scenarios. For adiabatic models, the peak $\ell$ values follow the series $(1: 2: 3: 4 \ldots)$, whereas for isocurvature models $(1: 3: 5: 7 \ldots)$. Since the first peak is contaminated by the early ISW effect (see Fig. 21), the higher peaks are the most reliable measure of this effect. On the other hand, this rise to the first peak can also be used to separate isocurvature from 
adiabatic models. We have noted in $\S$ IVC that the first isocurvature oscillation is low in amplitude. Only in adiabatic models does the first oscillation truly stand out as a peak.

Once adiabatic and isocurvature models are distinguished, the location of the peaks is uniquely predicted by the cosmological parameters. However, the degeneracy in the dependence on $\Omega_{0} h^{2}, \Omega_{\Lambda} h^{2}, h$, and $\Omega_{b} h^{2}$ does not allow inversion of the relation [47]. For example, an $\Omega_{b}=\Omega_{0}=1, h=0.5$ adiabatic model predicts $\ell \sim 400$ and $h=1.0, \ell \sim 500$ which can mimic projection effects from curvature and $\Lambda$. Of course, if one is willing to restrict $\Omega_{b} h^{2}$ to lie within the nucleosynthesis bounds, its effect on $r_{s}\left(\eta_{*}\right)$ is negligible. On the other hand, the geodesic deviation due to $K$ with low $\Omega_{0}$ and $\Lambda=0$ is a severe and easily tested effect. If the angular location of the peaks turn out to be multiples of a high $\ell \gtrsim 400-500$, then curvature must almost certainly be present in the model since no reasonable change in $r_{s}\left(\eta_{*}\right)$ or $\eta_{0}-\eta_{*}$ can account for it [48].

Since isocurvature acoustic oscillations are likely to be erased by reionization, let us concentrate on lifting the degeneracy for the more plausible adiabatic case. We can use the deviation of the first peak from the acoustic series predicted above for this purpose. The early ISW effect pushes the peak to larger scales for low $\Omega_{0} h^{2}$ universes. Moreover, the amplitudes of the peaks contain a large amount of cosmological information as well. Even though this depends on the underlying power spectrum, a minimal assumption, such as a pure power law only over the range of the peaks, would be sufficient to allow interesting constraints on cosmological parameters. As we have seen,

(a) Lowering $\Omega_{0} h^{2}$ boosts the first peak relative to the higher peaks due to early ISW contributions

(b) Raising $\Omega_{b} h^{2}$ boosts the odd numbered peaks over the even due to reduction in the pressure relative to the gravitational force.

In fact, these opposing $h$ dependences nearly cancel for the first peak if $\Omega_{0}=1$ and $\Omega_{b} h^{2}$ is given by big bang nucleosynthesis. This is not true for the higher peaks [6,32]. Thus the relative amplitudes of the series of peaks contain crucial cosmological information. These important tests will depend on having experimental information for anisotropies $\ell \gtrsim 200$.

Considering the present experimental focus on $\ell \lesssim 200$ anisotropies, it would be useful to extract information from the ratio of large to intermediate angle anisotropies. For instance, in the $n=1$ model of Fig. 21a, the rise to the first peak is more dramatic in low $\Omega_{0} h^{2}$ universes. Unfortunately, this of course depends on the specific model in question. However in general, lowering $\Omega_{0} h^{2}$ increases the intermediate anisotropies through the early ISW effect whereas increasing $\Omega_{b} h^{2}$ does the same through the acoustic oscillations. For large scales, the late ISW effect can boost anisotropies a comparable amount in the open but not the $\Lambda$ case. However one must recall that in the open case there is also Poisson suppression of the power spectrum and other curvature effects.

Of course, allowing the thermal history to deviate from the standard recombination scenario introduces another degree of freedom which complicates the extraction of cosmological information. If reionization is low, the acoustic peaks which are damped as $e^{-\tau}$ below the horizon at last scattering, may still be observable. However if the ionization is high, the detailed information in the acoustic oscillations is lost to us. This is likely to be the case for isocurvature models. If the initial spectrum is chosen to be consistent with large scale structure $n \simeq-1$, the large fluctuations at small scales could result in reionization. Normalized to large scale anisotropies, standard recombination models also produce excessively large intermediate scale 
adiabatic oscillations in the standard recombination scenario (see Fig. 21b). Reionization is therefore also necessary.

In this case the sole feature is the damping scale which measures the photon diffusion length at last scattering. In Fig. 21b, we show the effects of altering the ionization history of open and $\Lambda$ isocurvature models. Assuming a cosmological model, the damping scale fixes the ionization history. On the other hand, assuming an ionization history (e.g. fully ionized), it essentially probes the horizon size at last scattering as projected via geodesic deviation. Although $\Lambda$ models are older and yield a larger distance to the last scattering surface, the geodesic deviation effect pushes the damping scale of open models to even smaller angles. Notice that this also makes the open universe large angle anisotropies nearly independent of ionization history since these angles correspond to superhorizon scales at last scattering.

As for the amplitude of the regenerated fluctuations, we may employ the analysis of $\S \mathrm{VB}$, to gain insight into the numerical results. In Fig. 22, we show a comparison of isocurvature temperature power spectra from the numerical and analytical calculations. The numerical calculations are purely first order and do not include the Vishniac contribution. The Doppler and SW (DSW) fluctuations are increasingly suppressed by thickness cancellation as last scattering is delayed, as reflected in the time integrals of equation (58). The late ISW effect is of course independent of ionization but increases as $\Omega_{0}$ decreases. For the fully ionized, low $\Omega_{0}=0.1$ universe shown here, the late ISW contribution thus more than doubles the temperature fluctuations at intermediate scales. On the other hand, the Vishniac effect depends quadratically on the amplitude of the matter fluctuations and thus is larger for later last scattering. In our detailed numerical study [4], we show how these various effects can be combined to yield the minimal anisotropies for the isocurvature model.

Reionized adiabatic models look similar to isocurvature models in that the sole feature is at the diffusion scale at last scattering. If no underlying power spectrum is assumed, it may be difficult to distinguish between the two. However, as large scale structure measurements reach to larger scales and CMB experiments to smaller scales, it will be possible to entirely remove the ambiguity of the initial power spectrum (see e.g. [49]). Consistency between the matter and radiation power spectrum is indeed the ultimate test of any model for structure formation. As we have seen, the difference in the matter and temperature transfer functions on the same scale can remove all doubt on the question of adiabatic vs. isocurvature initial conditions and/or standard recombination vs. reionized thermal histories.

\section{Discussion}

We have comprehensively studied the evolution of density and temperature perturbations with an arbitrary spectrum of adiabatic and isocurvature perturbations in a critical $\Omega_{0}=1$, open, and $\Lambda$ dominated expanding universe. By employing an analytic treatment, we provide model independent insight into the formation of anisotropies that is confirmed by its agreement with the full numerical calculation. It thus becomes possible to separate and interpret each physical process that generates these perturbations.

Our treatment identifies numerous sources of anisotropies. Curvature effects due to geodesic deviation and on the fluctuations themselves give rise to peculiarities in the anisotropy spectrum which may soon be constrained by the observations. Moreover gravitational redshift effects due to the photon's climb out of the potential well (SW effect) as well as decay or growth in the potential due to radiation (early ISW effect) and the decay due to the rapid expansion in an open or $\Lambda$ dominated universe (late ISW effect) carry specific 
signatures that may be identifiable in the large angle anisotropies. However, the manifestation of these effects in a particular model will depend on the initial power spectrum. In examining the dependence on initial conditions, we also present a particularly simple derivation of the $\frac{1}{3}$ (adiabatic) and 2 (isocurvature) coefficients multiplying the gravitational potential in the SW effect.

Smaller angle anisotropies carry information which is less dependent on the power spectrum. We have investigated the nature of acoustic oscillations which give rise to peaks in the anisotropy as well as diffusion damping which is responsible for its small angle cutoff. Moreover, we have provided a very simple formula which predicts the angular location of the peaks as a function of the matter content and geometry of the universe. The physical origin of their relative heights is also clarified. In reionized models however, acoustic oscillations are damped and give way to last scattering effects due to baryons in infall. At intermediate scales, this leads to the Doppler effect whereas at small scales significant second order Vishniac contributions must be considered.

Although the principles outlined here are valid for any model, they can also be used to evaluate currently popular models for structure formation. At the present however, it is not even clear which model, if any, is consistent with the large scale structure data alone, much less the detailed features in the CMB anisotropies. Despite the success of the elegantly simple standard CDM model for structure formation, it is becoming increasingly clear that some modification either in the model or our understanding of its implications is necessary (e.g. see [50] for a review). Normalized to large scale anisotropies, standard CDM predicts matter fluctuations which imply a moderately anti-biased picture of galaxy formation [51] and more small scale power than is observed for peculiar velocities. It is also difficult to understand the dynamical measurements of a low $\Omega_{0}$ at small scales in this picture [52]. The obvious solutions within the context of CDM are to either change the initial power spectrum from Harrison-Zel'dovich $n=1$, or lower $\Omega_{0}$ to move the equality cut off to larger scales. Indeed the shape of the matter power spectrum alone seems to indicate $\Omega_{0} h \simeq 0.25$ [43], and determinations of a high Hubble constant $h \simeq 0.7-0.8$, if confirmed, also support low $\Omega_{0}$ models due to the age problem [53].

We have fully examined the consequences for anisotropies of these standard solutions. The signature of low $\Omega_{0}$ models at large scales depends on the underlying power spectrum. Particularly in the case of open models, where we expect deviations from a single power law spectrum at the curvature scale, this ambiguity can change the relative amplitudes of anisotropies to matter fluctuations as well as the shape of the large scale anisotropies themselves. For $\Lambda$ models, this is perhaps less of a concern. The boost in low order multipoles from the late ISW effect can be used to constrain $n=1$ models [54]. The acoustic peaks provide a better handle on the underlying cosmology from both their angular location and relative heights. Even with complications such as gravitational wave contributions, which can boost the large scale anisotropy relative to the matter [55], the information contained in the acoustic peaks is not lost.

Another possible alternative is to abandon adiabatic fluctuations in favor of isocurvature ones. This model also changes the relative amplitude of matter versus temperature perturbations. However given the likelihood of reionization, the thermal history of baryonic isocurvature models can be adjusted to match the observations. The fundamental probe here is the slope of the matter and temperature power spectra. Present indications are that $n \simeq-1\left(n_{\text {eff }}=2\right)$ from large scale structure measurements. The implied discrepancy with flat large scale anisotropies with $n_{\text {eff }} \simeq 1$ [56] is beginning to indicate that no single power 
law model is adequate [26]. While this is not necessarily surprising for the open version, it would require a dramatic break in the power spectrum to counter the heavily small scale weighted power required by large scale structure. Perhaps more damaging to this model is the growing body of intermediate scale $\ell \simeq 50-200$ anisotropy measurements. If a steep rise toward $\ell \simeq 200$ is also confirmed [57], there will also have to be an additional break below the curvature scale. Furthermore, there are indications that even large scale structure measurements themselves do not fit with single initial power law isocurvature models due to features in the matter transfer function [43].

Finally a change in the matter content, e.g. adding massive neutrinos [58] or topological defects [59], is another possibility. Although we do not explicitly consider such exotic models, the principles outlined here remain valid. Sachs-Wolfe contributions and acoustic oscillations are determined from the gravitational potential in the same way in these models. Thus once the evolution of the matter is understood, the implications for anisotropies is apparent.

Given that none of these alternatives provide a compelling $a b$ initio model for structure formation, it is perhaps best to keep an open mind to all of these possibilities. As the large scale structure and CMB anisotropy data continue to accumulate, the general principles formulated here will aid in the empirical reconstruction of a consistent model for structure formation.

Many are those under heaven who attend to their theories and techniques,
and they all believe that nothing can be added to the ones they possess.
Where is the true way of old to be found?
\[ \begin{array}{l}\text { In a haze! Where am I going? } \\ \text { In a daze! Where shall I arrive? } \\ \text { With the myriad things before me, } \\ \text { None will serve as final destination. }\end{array} \]

-Chuang-tzu

\section{Acknowledgements}

We would like to thank D. Scott, J. Silk, M. White, and anyone with the patience to read this far! W.H. acknowledges support from the NSF and N.S. from a JSPS fellowship. 


\section{References}

[1] M. White, D. Scott, and J. Silk, Ann. Rev. Astron. Astrophys., 32, 319 (1994).

[2] P.J.E. Peebles, Astrophys. J. Lett., 315, L73 (1987); P.J.E. Peebles, Nature, 327, 210 (1987).

[3] G. Efstathiou and J.R. Bond, Mon. Not. Roy. Astron. Soc., 227, 33p (1987).

[4] W. Hu and N. Sugiyama, Astrophys. J., (in press).

[5] N. Sugiyama and J. Silk, Phys. Rev. Lett, 73, 509 (1994).

[6] W. Hu and N. Sugiyama, Astrophys. J., (submitted 1994).

[7] A.G. Doroshkevich, Ya. B. Zel'dovich, R.A. Sunyaev, Sov. Astron, 22, 523 (1978).

[8] H. Kodama and M. Sasaki, Int. J. Mod. Phys., A1, 265 (1986).

[9] R.K. Sachs and A.M. Wolfe, Astrophys. J., 162, 815 (1970).

[10] M.L. Wilson, Astrophys. J., 273, 2 (1983).

[11] J.R. Bond and G. Efstathiou, Mon. Not. Roy. Astron. Soc., 226, 665 (1987); J.R. Bond, in The Early Universe, eds. W.G. Unruh and G.W. Semenoff, (Dordrecht, Boston) p. 283.

[12] J. Silk, Astrophys. J., 151, 459 (1968).

[13] R.A. Sunyaev and Ya. B. Zel'dovich, Astrophys. Sp. Sci., 7, 3 (1970).

[14] J.M. Bardeen, Phys. Rev., D22, 1882 (1980). Note his $\Phi_{H}=\Phi$ and $\Phi_{A}=\Psi$.

[15] H. Kodama and M. Sasaki, Prog. Theor. Phys. Suppl., 78, 1 (1984).

[16] V.F. Mukhanov, H.A. Feldman, and R.H Brandenberger, Phys. Rep., 215, 203 (1992).

[17] N. Gouda, M. Sasaki, Y. Suto, Astrophys. J., 341, 557 (1989).

[18] E.M. Liftshitz and I.M. Khalatnikov, Adv. Phys., 12, 185 (1963).

[19] E. R. Harrison, Phys. Rev., D1, 2726 (1970).

[20] L.F. Abbott and R.K. Schaefer, Astrophys. J., 308, 546 (1986). Their definition of the radial eigenfunctions is equivalent to our $M_{\ell}^{1 / 2} X_{\nu}^{\ell}$ (see also [22]).

[21] N. Gouda, N. Sugiyama, and M. Sasaki, Prog. Theor. Phys., 85, 1023 (1991).

[22] The stability problem can be avoided by two tricks: rewrite the Boltzmann equation with $\Delta_{\gamma}$ replacing $\Theta_{0}$ and $\Theta_{\ell}^{\prime}=M_{\ell}^{1 / 2} \Theta_{\ell}$ instead of $\Theta_{\ell}$.

[23] N.Y. Gnedin and J.P. Ostriker, Astrophys. J., 400, 1 (1992).

[24] R. Cen, J.P. Ostriker, and P.J.E. Peebles, Astrophys. J., 415, 423 (1993).

[25] N. Sugiyama and N. Gouda, Prog. Theor. Phys., 88, 803 (1992).

[26] W. Hu, in CWRU CMB Workshop: 2 Years after COBE, eds. L. Krauss \& P. Kernan, (World Scientific, Singapore), in press.

[27] W. Hu, N. Sugiyama, Phys. Rev., D50, 627 (1994).

[28] L. Kofman and A. Starobinskii, Sov. Astr. Lett, 11, 271 (1985).

[29] M. Kamionkowski and D. Spergel, Astrophys. J., 432, 7 (1994).

[30] H.E. Jørgensen, E. Kotok, P. Naselsky, and I. Novikov, Astron. Astrophys., (in press).

[31] F. Atrio-Barandela, and A.G. Doroshkevich, Astrophys. J., 420, 26 (1994).

[32] U. Seljak, Astrophys. J., (submitted 1994).

[33] P.J.E. Peebles and J.T. Yu, Astrophys. J., 162, 85 (1970).

[34] P.J.E. Peebles, Large Scale Structure of the Universe, (Princeton University, Princeton 1980). 
[35] R.A. Sunyaev and Ya. B. Zel'dovich, Astrophys. Sp. Sci., 9, 368 (1970).

[36] W. Hu, D. Scott, and J. Silk, Astrophys. J. Lett., 430, L5, (1994).

[37] B.J.T. Jones and R.F.G. Wyse, Astron. Astrophys., 149, 144 (1985).

[38] N. Kaiser, Astrophys. J., 282, 374 (1984).

[39] G. Efstathiou, Large Scale Motions in the Universe: A Vatican Study Week, eds. Rubin, V.C. and Coyne, G.V., (Princeton University, Princeton, 1988) pg. 299.

[40] J.P. Ostriker and E.T. Vishniac, Astrophys. J. 306, 51 (1986); E.T. Vishniac, Astrophys. J., 322, 597 (1987).

[41] W. Hu, D. Scott, and J. Silk, Phys. Rev., D49, 648 (1994).

[42] D.H. Lyth and E.D. Stewart, Phys. Lett., B252, 336 (1990); B. Ratra and P.J.E. Peebles, Astrophys. J. Lett., 432, L5 (1994).

[43] J.A. Peacock and S.J. Dodds, Mon. Not. Roy. Astron. Soc., 267, 1020 (1994).

[44] G. Efstathiou, J.R. Bond, and S.D.M. White, Mon. Not. Roy. Astron. Soc., 258, P1 (1992).

[45] T. Chiba, N. Sugiyama, Y. Suto, Astrophys. J., 429, 427 (1994).

[46] T. Suginohara and Y. Suto, Astrophys. J., 387, 431 (1992).

[47] J.R. Bond, et al., Phys. Rev. Lett, 72, 13, 1994.

[48] M. Kamionkowski, D.N. Spergel, and N. Sugiyama, Astrophys. J. Lett., 426, L57 (1994)

[49] K. Gorski, Astrophys. J. Lett., 370, L5 (1989); M. Tegmark, E. Bunn, and W. Hu, Astrophys. J., 434, 1 (1994).

[50] J.P. Ostriker, Ann. Rev. Astron. Astrophys., 31, 689 (1993).

[51] E. Bunn, D. Scott, and M. White, Astrophys. J. Lett, submitted (1994).

[52] A. Dekel, et al., Astrophys. J., 412, 1 (1993).

[53] G. Jacoby, et al., PASP, 104, 599 (1992).

[54] E. Bunn and N. Sugiyama, Astrophys. J. Lett, submitted (1994).

[55] M.S. Turner, M. White, J.E. Lidsey, Phys. Rev., D48, 4613 (1993); R. Crittenden et al., Phys. Rev. Lett., 71, 324 (1993).

[56] K. Gorski, et al., Astrophys. J. Lett., 430, L89 (1994)

[57] D. Scott and M. White, in CWRU CMB Workshop: 2 Years after COBE, eds. L. Krauss \& P. Kernan, (World Scientific, Singapore), in press.

[58] M. Davis, F.J. Summers, D. Schlegel, Nature, 359, 393 (1992); A. Klypin, J. Holtzman, J. Primack, E. Regos, Astrophys. J., 416, 1 (1993)

[59] U.-L. Pen, D.N. Spergel, and N. Turok, Phys. Rev., D49, 692 (1994). 


\section{Appendix A: Open Universe Normal Modes}

\section{The Radial Representation}

Fluctuations in an open universe must be decomposed in the eigenfunctions of the Laplacian $\gamma^{i j} Q_{\mid i j}=$ $-k^{2} Q$. To gain intuition about these functions, let us examine an explicit representation. In radial coordinates the 3 -metric becomes

$$
\gamma_{i j} d x^{i} d x^{j}=-K^{-1}\left[d \chi^{2}+\sinh ^{2} \chi\left(d \theta^{2}+\sin ^{2} \theta d \phi^{2}\right)\right],
$$

where recall $\chi=\sqrt{-K} \eta$. Curvature makes the surface area of a shell at distance $\eta$ increase as $-K^{-1} e^{2 \chi}$ rather than $\eta^{2}$ for super-curvature distances $\chi \gg 1$. The Laplacian can now be written as

$$
\gamma^{i j} Q_{\mid i j}=-K \sinh ^{-2} \chi\left[\frac{\partial}{\partial \chi}\left(\sinh ^{2} \chi \frac{\partial Q}{\partial \chi}\right)+\sin ^{-1} \theta \frac{\partial}{\partial \theta}\left(\sin \theta \frac{\partial Q}{\partial \theta}\right)+\sin ^{-2} \theta \frac{\partial^{2} Q}{\partial \phi^{2}}\right] .
$$

Since the angular part is independent of curvature, we may separate variables such that $Q=X_{\nu}^{\ell}(\chi) Y_{\ell}^{m}(\theta, \phi)$ where $\nu^{2}=\tilde{k}^{2} /(-K)=-\left(k^{2} / K+1\right)$. From equation (A-2), it is obvious that the spherically symmetric $\ell=0$ function is

$$
X_{\nu}^{0}(\chi)=\frac{\sin (\nu \chi)}{\nu \sinh \chi}=\sqrt{-K} \frac{\sin (\tilde{k} \Delta \eta)}{\tilde{k} \sinh (\Delta \eta \sqrt{-K})} .
$$

As expected, the change in the area element from a flat to curved geometry causes $\sqrt{-K} \eta \rightarrow \sinh \chi$ in the denominator. The higher modes are explicitly given by $[18,19]$

$$
X_{\nu}^{\ell}(\chi)=(-1)^{\ell+1} M_{\ell}^{-1} \nu^{-2}\left(\nu^{2}+1\right)^{-\ell / 2} \sinh ^{\ell} \chi \frac{d^{\ell+1}(\cos \nu \chi)}{d(\cosh \chi)^{\ell+1}}
$$

and becomes $j_{\ell}(k \Delta \eta)$ in the flat space limit. Here

$$
M_{\ell}(\tilde{k}) \equiv \frac{\left(\tilde{k}^{2}-K\right) \ldots\left(\tilde{k}^{2}-K \ell^{2}\right)}{\left(\tilde{k}^{2}-K\right)^{\ell}},
$$

which reduces to unity as $K \rightarrow 0$. It represents our convention for the normalization of the open universe functions.

It is often more convenient to generate these functions from their recursion relations [20]. One such recursion relation is

$$
\frac{d}{d \eta} X_{\nu}^{\ell}=\frac{\ell}{2 \ell+1} k X_{\nu}^{\ell-1}+\frac{\ell+1}{2 \ell+1}\left[1-\ell(\ell+2) \frac{K}{k^{2}}\right] k X_{\nu}^{\ell+1},
$$

which is of the same form as the Boltzmann equation (6) for $(\Theta+\Psi) /(2 \ell+1)$ in the free streaming limit. This is quite natural since free streaming photons arrive at the observer on radial geodesics as an examination of equation ( $\mathrm{A}-1)$ shows. Thus the solution of the free streaming Boltzmann equation in the absence of the ISW term is obvious:

$$
\frac{\Theta_{\ell}(\eta, \tilde{k})}{2 \ell+1}=\left[\Theta_{0}+\Psi\right]\left(\eta_{*}, \tilde{k}\right) X_{\nu}^{\ell}\left(\chi-\chi_{*}\right)
$$

where we have assumed that the boundary condition at last scattering is given by the monopole fluctuation as is appropriate to the SW effect. The ISW effect acts like an impulse $(\dot{\Psi}-\dot{\Phi}) \delta \eta$ at some intermediate time $\eta$ which then free streams to the present. The full solution therefore is

$$
\frac{\Theta_{\ell}(\eta, \tilde{k})}{2 \ell+1}=\left[\Theta_{0}+\Psi\right]\left(\eta_{*}, \tilde{k}\right) X_{\nu}^{\ell}\left(\chi-\chi_{*}\right)+\int_{\eta_{*}}^{\eta}[\dot{\Psi}-\dot{\Phi}]\left(\eta^{\prime}, \tilde{k}\right) X_{\nu}^{\ell}\left(\chi-\chi^{\prime}\right) d \eta^{\prime} .
$$


Let us now examine the peculiar nature of the eigenfunctions. Since they are complete for $k \geq \sqrt{-K}$, i.e. $\tilde{k} \geq 0$, should $2 \pi / k$ or $2 \pi / \tilde{k}$ be considered the effective wavelength? In Fig. 23 , we plot the spherically symmetric $\ell=0$ mode given by equation (A-3). The argument in favor of $\tilde{k}$ is that its first zero is at $\Delta \eta=\pi / \tilde{k}$. This is related to the completeness property: the zero crossing property shows that as $\tilde{k} \rightarrow 0$ we can obtain arbitrarily large structures. However even in this limit, the amplitude of the structure above the curvature scale is suppressed as $e^{-\chi}$. The effective scale of the prominent structure thus goes to the curvature scale favoring $k^{-1}=1 / \sqrt{-K}$ as the effective wavelength. In fact, the $e^{-\chi}$ behavior is independent of the wavenumber and $\ell$, if $\chi \gg 1$.

This peculiarity in the eigenmodes has significant consequences. Any random phase superposition of the eigenmodes $X_{\nu}^{\ell}$ will have exponentially suppressed structure larger than the curvature radius. Even though completeness tells us that arbitrarily large structure can be built out of the $X_{\nu}^{\ell}$ functions, it cannot be done without correlating the modes. This is even if the structure has support only to a finite radius which is above the curvature scale.

Is the random phase hypothesis and the lack of structure above the curvature scale reasonable? The fundamental difference between open and flat universes is that the volume increases exponentially with the radial coordinate above the curvature scale $V\left(\chi_{c}\right) \sim\left[\sinh \left(2 \chi_{c}\right)-2 \chi_{c}\right]$ as the line element of equation (A-1) shows. Structure above the curvature scale implies correlations over vast volumes [29]. It is in fact difficult to conceive of a model where correlations do not die exponentially above the curvature radius. The random phase hypothesis has been proven to be valid for adiabatic inflationary perturbations [42]. However, a definitive answer to this question for isocurvature models awaits the invention of a mechanism for generating such perturbations in a consistent model for structure formation.

\section{General Angular Functions}

Although the radial representation suffices for many purposes, often one needs the full machinery of the general normal mode decomposition. Formally, the angular and spatial fluctuations of the full radiation field is decomposed into [10]

$$
\Theta(\eta, \mathbf{x}, \gamma)=\sum_{\ell=0}^{\infty} \Theta_{\ell}(\eta, k) G_{\ell}(\mathbf{x}, \gamma)
$$

where

$$
G_{\ell}(\mathbf{x}, \gamma)=(-k)^{-\ell} Q_{\mid i_{1} \ldots i_{\ell}}(\mathbf{x}) P_{\ell}^{i_{1} \ldots i_{\ell}}(\mathbf{x}, \gamma)
$$

and

$$
\begin{aligned}
P_{0} & =1, \quad P_{1}^{i}=\gamma^{i}, \\
P_{2}^{i j} & =\frac{1}{2}\left(3 \gamma^{i} \gamma^{j}-\gamma^{i j}\right), \\
P_{\ell+1}^{i_{1} \ldots i_{\ell+1}} & =\frac{2 \ell+1}{\ell+1} \gamma^{\left(i_{1}\right.} P_{\ell}^{\left.i_{2} \ldots i_{\ell+1}\right)}-\frac{\ell}{\ell+1} \gamma^{\left(i_{1} i_{2}\right.} P_{\ell-1}^{\left.i_{3} . i_{\ell+1}\right)},
\end{aligned}
$$

with parentheses denoting symmetrization about the indices. For flat space, this becomes $G_{\ell}=(-i)^{\ell} \exp (i \mathbf{k}$. $\mathbf{x}) P_{\ell}(\mathbf{k} \cdot \boldsymbol{\gamma})$, where $P_{\ell}$ is an ordinary Legendre polynomial. Notice that along a path defined by fixed $\boldsymbol{\gamma}$, the flat $G_{\ell}$ becomes $j_{\ell}(k \eta)$ after averaging over $k$-directions. Travelling on a fixed direction away from a point is the same as following a radial path outwards. Thus fluctuations along this path can be decomposed in 
the radial eigenfunction. We shall see that this argument can be generalized to the open universe case and allows one to interpret equation (A-10) more easily.

We can also use the properties of $G_{\ell}$ to simplify the Boltzmann equation (3). The anisotropic stress perturbation of the photons, defined as

$$
\Pi_{\gamma}^{i j} \equiv 4 \int \frac{d \Omega}{4 \pi}\left(\gamma^{i} \gamma^{j}-\frac{1}{3} \gamma^{i j}\right) \Theta(\eta, \mathbf{x}, \gamma)
$$

is therefore related to the quadrupole moment,

$$
\frac{1}{16} \gamma_{i} \gamma_{j} \Pi_{\gamma}^{i j}=\frac{1}{10} \Theta_{2} G_{2}
$$

The recursion relation

$$
\begin{aligned}
\gamma^{i} G_{\ell \mid i} & =\frac{d}{d \eta} G[\mathbf{x}(\eta), \gamma(\eta)]=\dot{x}^{i} \frac{\partial}{\partial x^{i}} G_{\ell}+\dot{\gamma}^{i} \frac{\partial}{\partial \gamma^{i}} G_{\ell} \\
& =k\left\{\frac{\ell}{2 \ell+1}\left[1-\left(\ell^{2}-1\right) \frac{K}{k^{2}}\right] G_{\ell-1}-\frac{\ell+1}{2 \ell+1} G_{\ell+1}\right\},
\end{aligned}
$$

which follows from equation (A-10) and (A-11) [21], completes the simplification of equation (3) to (6). Here we take $\mathbf{x}(\eta)$ to be the integral path along $\gamma$. By comparing equations (A-6) and (A-14), the open universe generalization of the relation between $G_{\ell}$ and the radial eigenfunction is now apparent:

$$
G_{\ell}[\mathbf{x}(\eta), \gamma(\eta)]=M_{\ell} X_{\nu}^{\ell}(\eta)
$$

The only conceptual difference is that for the radial path that we decompose fluctuations on, $\gamma$ is not constant. This also clarifies the interpretation of the recursion relation for $G_{\ell}$ [equation (A-14)]. Finally by employing these definitions, we may write the temperature correlation function as [10]

$$
\left\langle\Theta^{*}\left(\eta_{0}, \mathbf{x}, \gamma\right) \Theta\left(\eta_{0}, \mathbf{x}, \gamma^{\prime}\right)\right\rangle=\frac{V}{2 \pi^{2}} \int \frac{d \tilde{k}}{\tilde{k}} \sum_{\ell} \frac{M_{\ell}(\tilde{k})}{2 \ell+1} \tilde{k}^{3}\left|\Theta_{\ell}\left(\eta_{0}, \tilde{k}\right)\right|^{2} P_{\ell}\left(\boldsymbol{\gamma} \cdot \boldsymbol{\gamma}^{\prime}\right),
$$

where $P_{\ell}$ is a Legendre polynomial. This implies the definition of $C_{\ell}$ in equation (8).

\section{Appendix B. Single Fluid and Other Useful Relations}

Above the horizon the entropy perturbation $S$ is constant, and all perturbation quantities can be obtained from the solution for the total density perturbation $\Delta_{T}$. Combining the total continuity and Euler equations in (16) yields the second order evolution equation

$$
\left\{\frac{d^{2}}{d a^{2}}-\frac{f}{a} \frac{d}{d a}+\frac{1}{a^{2}}\left[\left(\frac{k}{k_{e q}}\right)^{2}\left(1-\frac{3 K}{k^{2}}\right) h-g\right]\right\} \Delta_{T}=\left(\frac{k}{k_{e q}}\right)^{2}\left(1-\frac{3 K}{k^{2}}\right) j S
$$

where

$$
\begin{aligned}
f & =\frac{3 a}{4+3 a}-\frac{5}{2} \frac{a}{1+a}, \\
g & =2+\frac{9 a}{4+3 a}-\frac{a}{2} \frac{6+7 a}{(1+a)^{2}} \\
h & =\frac{8}{3} \frac{a^{2}}{(4+3 a)(1+a)}, \\
j & =\frac{8}{3} \frac{a}{(4+3 a)(1+a)^{2}},
\end{aligned}
$$


where recall that $a$ is normalized to unity at matter-radiation equality. Here we have taken the anisotropic stress $\Pi=0$ and assumed that the universe is in the matter or radiation dominated epoch. The solutions to the homogeneous equation with $S=0$ are given by

$$
\begin{aligned}
U_{A} & =\left[a^{3}+\frac{2}{9} a^{2}-\frac{8}{9} a-\frac{16}{9}+\frac{16}{9} \sqrt{a+1}\right] \frac{1}{a(a+1)}, \\
U_{D} & =\frac{1}{a \sqrt{a+1}},
\end{aligned}
$$

and represent the growing and decaying mode of adiabatic perturbations respectively. Using Green's method, the particular solution in the presence of a constant entropy fluctuation $S$ becomes $\Delta_{T}=C_{A} U_{A}+C_{D} U_{D}+$ $S U_{I}$, where $U_{I}$ is given by

$$
U_{I}=\frac{4}{15}\left(\frac{k}{k_{e q}}\right)^{2}\left(1-\frac{3 K}{k^{2}}\right) \frac{3 a^{2}+22 a+24+4(4+3 a)(1+a)^{1 / 2}}{(1+a)(3 a+4)\left[1+(1+a)^{1 / 2}\right]^{4}} a^{3} .
$$

After radiation becomes negligible, the both isocurvature and adiabatic modes evolve in the same manner

$$
\ddot{\Delta}_{T}+\frac{\dot{a}}{a} \dot{\Delta}_{T}=4 \pi G \rho\left(\frac{a}{a_{0}}\right)^{2} \Delta_{T}
$$

For pressureless perturbations, each mass shell evolves as a separate homogeneous universe. Since a density perturbation can be viewed as merely a different choice of the initial time surface, the evolution of the fractional shift in the scale factor, i.e. the Hubble parameter $H$, must coincide with $\Delta_{T}$. It is simple to check that the Friedman equations do indeed imply

$$
\ddot{H}+\frac{\dot{a}}{a} \dot{H}=4 \pi G \rho\left(\frac{a}{a_{0}}\right)^{2} H,
$$

so that one solution, the decaying mode, of equation (B-5) is $\Delta_{T} \propto H$ [34]. The growing mode $\Delta_{T} \propto D$ can easily be determined by writing its form as $D \propto H G$ yielding

$$
\ddot{G}+\left(\frac{\dot{a}}{a}+2 \frac{\dot{H}}{H}\right) \dot{G}=0
$$

which can be immediately solved as [34]

$$
D(a) \propto H \int \frac{d a}{(a H)^{3}}
$$

Note that we ignore pressure contributions in $H$ [c.f. equation (20)]. If the cosmological constant $\Lambda=0$, this integral can be performed analytically

$$
D(a) \propto 1+\frac{3}{x}+\frac{3(1+x)^{1 / 2}}{x^{3 / 2}} \ln \left[(1+x)^{1 / 2}-x^{1 / 2}\right]
$$

where $x=\left(\Omega_{0}^{-1}-1\right)\left(a / a_{0}\right)$. In the more general case, a numerical solution to this integral must be employed. Since before curvature or $\Lambda$ domination $D \propto a$, the full solution for $\Delta_{T}$, where the universe is allowed to pass through radiation, matter and curvature or $\Lambda$ domination, can be simply obtained from equation (B-3) and (B-4), by replacing $a$ with $D$ normalized so that $D=a$ early on. 
With the solution for $\Delta_{T}$ and the definition of $S$ [equation (21)], all component perturbations can be written in terms of $\Delta_{T}$. For example, in the baryonic isocurvature scenario,

$$
\Delta_{b}=\frac{1}{4+3 a}\left[4 S+3(1+a) \Delta_{T}\right]
$$

and

$$
\begin{aligned}
\Delta_{\nu} & =\frac{4}{3}\left(\Delta_{b}-S_{b \nu}\right), \\
\Delta_{\gamma} & =\frac{4}{3}\left(\Delta_{b}-S_{b \gamma}\right) .
\end{aligned}
$$

The fact that in this model the curvature perturbation vanishes initially when the universe is radiation dominated allows us to set $S_{b \nu}=S_{b \gamma}$. The velocity and potentials can be written as

$$
\begin{aligned}
V_{T} & =-\frac{3}{k} \frac{\dot{a}}{a}\left(1-\frac{3 K}{k^{2}}\right)^{-1} \frac{1+a}{4+3 a}\left[a \frac{d \Delta_{T}}{d a}-\frac{1}{1+a} \Delta_{T}\right], \\
\Psi & =-\frac{3}{4}\left(\frac{k_{e q}}{k}\right)^{2}\left(1-\frac{3 K}{k^{2}}\right)^{-1} \frac{1+a}{a^{2}} \Delta_{T},
\end{aligned}
$$

where note that constant entropy assumption requires that all the velocities $V_{i}=V_{T}$. The relation for the velocity may be simplified by noting that

$$
\begin{aligned}
\eta(a) & \simeq \frac{2 \sqrt{2}}{k_{e q}}[\sqrt{1+a}-1] \quad \mathrm{RD} / \mathrm{MD} \\
& \simeq \frac{1}{\sqrt{-K}} \cosh ^{-1}\left[1+\frac{2\left(1-\Omega_{0}\right)}{\Omega_{0}} \frac{a}{a_{0}}\right], \quad \mathrm{MD} / \mathrm{CD}
\end{aligned}
$$

where CD denotes curvature domination with $\Lambda=0$. For $\Lambda \neq 0$, it must be evaluated by numerical integration. Before curvature or $\Lambda$ domination

$$
\frac{\dot{a}}{a}=\frac{(1+a)^{1 / 2}}{\sqrt{2} a} k_{e q}
$$

which can be used to explicitly evaluate (B-12). Finally, in Tab. 1 we list some commonly used symbols in the paper and the equation in which they first appeared.

Table 1. Commonly used symbols. Time variables $a, z, \eta$, and $\chi$ are often used interchangably with special epochs listed here under scale factor $a$ entries. Component density $\Delta_{i}$ and velocity $V_{i}$ are defined in $\S \mathrm{IIC}$ and $\mathrm{D}$, with $i$ as $b$ for baryons, $\gamma$ for photons, $\nu$ for neutrinos, and $c$ for collisionless cold dark matter. Note that $V_{\gamma}=\Theta_{1}$ (see following page). 


\begin{tabular}{|c|c|c|}
\hline Symbol & Definition & Equation \\
\hline$\Delta_{T}$ & Total density fluctuation & $\overline{(11)}$ \\
\hline$\Theta$ & CMB temperature fluctuation & $\overline{(3)}$ \\
\hline$\Theta_{0}$ & CMB monopole fluctuation & (6) \\
\hline$\Theta_{\ell}$ & CMB $\ell$ th multipole fluctuation & $(6)$ \\
\hline$\Pi$ & Anisotropic stress perturbation & $(12)$ \\
\hline$\Psi$ & Gravitational (Newtonian) potential & $(13)$ \\
\hline$\Phi$ & Gravitational (curvature) potential & $(13)$ \\
\hline$\Psi$ & Gravitational (curvature) potential & $(13)$ \\
\hline$\eta$ & Conformal time & $(1)$ \\
\hline$\nu$ & Curvature normalized wavenumber & $(31)$ \\
\hline$\sigma_{T}$ & Thomson cross section & $(3)$ \\
\hline$\tau$ & Thomson optical depth & $(3)$ \\
\hline$\chi$ & Curvature normalized distance & $(31)$ \\
\hline $\mathcal{D}$ & Diffusion damping factor & $(39)$ \\
\hline $\mathcal{G}$ & Drag growth factor & $(52)$ \\
\hline$C_{A}$ & Initial adiabatic spectrum & $(17)$ \\
\hline$C_{I}$ & Initial isocurvature spectrum & $(17)$ \\
\hline$C_{\ell}$ & Anisotropy power spectrum & $(8)$ \\
\hline$D$ & Pressureless growth factor & $(19)$ \\
\hline$F$ & Gravitational driving force & $(34)$ \\
\hline$H$ & Hubble parameter & $(2)$ \\
\hline$\overline{N_{\ell}}$ & Neutrino $\ell$ th multipole & $(6)$ \\
\hline$K$ & Curvature & $(1)$ \\
\hline$R$ & Normalized scale factor $3 \rho_{b} / 4 \rho_{\gamma}$ & $(14)$ \\
\hline$S$ & Entropy fluctuation & $(16)$ \\
\hline$T$ & Matter transfer function & $(59)$ \\
\hline$V_{T}$ & Total velocity amplitude & $(11)$ \\
\hline$X_{\nu}^{\ell}$ & Radial eigenfunction & $(\mathrm{A}-3)$ \\
\hline$a$ & Scale factor & $(1)$ \\
\hline$a_{0}$ & Present scale factor & $(1)$ \\
\hline$a_{d}$ & Compton drag epoch & $(15)$ \\
\hline$a_{e q}$ & Equality scale factor & $(1)$ \\
\hline$a_{i}$ & Ionization epoch & $(53)$ \\
\hline$a_{*}$ & Recombination conformal time & $(7)$ \\
\hline$c_{s}$ & Photon-baryon sound speed & $(33)$ \\
\hline$k$ & Laplacian wavenumber & $(4)$ \\
\hline$\tilde{k}$ & Renormalized wavenumber & $(4)$ \\
\hline$k_{D}$ & Diffusion damping wavenumber & $(40)$ \\
\hline$k_{e q}$ & Equality horizon wavenumber & $(18)$ \\
\hline $\bar{\ell}$ & Multipole number & $(5)$ \\
\hline$r_{\theta}$ & Projection factor & $(51)$ \\
\hline$r_{s}$ & Sound horizon & $(36)$ \\
\hline$x_{e}$ & Electron ionization fraction & $(3)$ \\
\hline
\end{tabular}




\section{Figure Captions:}

Figure 1. Large scale open isocurvature evolution $\left(\Omega_{0}=0.2, h=0.5\right.$, no recombination). Perturbations, which originate in the baryons, are transferred to the radiation as the universe becomes more matter dominated to avoid a significant curvature perturbation. Nonetheless, radiation fluctuations create total density fluctuations from feedback. These adiabatic fluctuations in $\Delta_{T}$ dominate over the original entropy perturbation near horizon crossing $a_{H}$ in the matter dominated epoch. The single fluid approximation cannot extend after last scattering for the photons $a_{*}$, since free streaming will damp $\Delta_{\gamma}$ away. After curvature domination the total density is prevented from growing and thus leads to decay in the gravitational potential $\Psi$.

Figure 2. The total Sachs-Wolfe effect $\left(\Omega_{0}=0.1, h=0.5\right.$, standard recombination). In the adiabatic case, temperature fluctuations are enhanced in gravitational wells such that $\Theta$ and $\Psi$ cancel, yielding $\Theta_{0}+\Psi=1 / 3 \Psi$ in the matter dominated epoch. For the isocurvature case, the ISW effect creates a net total of $\Theta_{0}+\Psi=2 \Psi$ reflecting the anticorrelated nature of radiation and total density fluctuations. After last scattering at $a_{*}$, this SW contribution (analytic only) collisionlessly damps from the monopole. The rms temperature fluctuations (numerical only) acquires contributions after $a_{*}$ from the ISW effect due to the radiation (early) and curvature or $\Lambda$ (late) contributions. These contributions are relatively more important for adiabatic models due to the partial cancellation of $\Theta_{0}$ and $\Psi$ at last scattering. Since $\Lambda$ domination can only have occurred comparatively recently, the late ISW effect is also less important in a $\Lambda$ compared to an open universe.

Figure 3. $\Omega_{0}=1$ adiabatic full photon spectrum (standard recombination). Shown here and in Figs. 4,6,7 is the contribution to the anisotropy per logarithmic $\tilde{k}$ and $\ell$ interval $(\Delta T / T)_{\ell k}^{2}$ [equation (9)] with equally spaced contours up to a cut off set to best display the features in question. The strong correlation between $\ell$ and $k$ merely reflects the projection of a scale on the last scattering surface to an angle on the sky. At $\log \ell \gtrsim 2$, SW contributions fall off and are replaced by the acoustic peaks (saturated here). The detailed structure can be traced to the radial eigenfunction $X_{\nu}^{\ell}(\chi)=j_{\ell}(x)$ which governs the projection and free streaming oscillations.

Figure 4. $\Lambda$ adiabatic photon spectrum $\left(\Omega_{0}=0.1, h=0.5\right.$, standard recombination). Unlike the $\Omega_{0}=1$ case, this scenario has significant contributions from after last scattering through the early and late ISW effect. (a) The early ISW effect arises if horizon crossing is near radiation domination, and projects onto a second ridge which is more prominent than the SW ridge at intermediate but not large angles. (b) After $\Lambda$ domination, the late ISW contributions come free streaming in from the monopole yielding a boost in the low order multipoles for a small range in $k$, due to cancellation with SW contributions at the largest scales and crest-trough cancellation at smaller scales. Scales depicted in Fig. 5a,b are marked here by dashed lines.

Figure 5. Analytic separation of adiabatic large angle anisotropies $\left(\Omega_{0}=0.1 h=0.5\right.$, standard recombination, arbitrary normalization). Scales are chosen to match the features in Fig. 4 and 6. $\Lambda$ models: (a) At the largest scales, e.g. here $k=10^{-4} \mathrm{Mpc}^{-1}$, the SW effect dominates over the late ISW effect due to projection. However since the potential decays, the late ISW effect partially cancels the SW effect if the mode is superhorizon sized at $\Lambda$ domination. (b) Intermediate scale peaks in Fig. 4 are due to the late ISW boost of the higher SW free streaming ridges. Open models: (c) The maximum scale corresponds to the curvature radius $k=\sqrt{-K}$. For this scale, the SW effect projects broadly in $\ell$ peaking near $\ell \sim 10$. For the late ISW effect, this scale projects onto the monopole and dipole near curvature domination thus leaving the ISW contributions to decrease smoothly with $\ell$. (d) At smaller scales, corresponding to the large ridge in Fig. 6, the late ISW effect projects onto $\ell \simeq 2-10$ and completely dominates leading to a rising spectrum of anisotropies. 
Figure 6. Open adiabatic photon spectrum $\left(\Omega_{0}=0.1, h=0.5\right.$, standard recombination). (a) Like the $\Lambda$ case, the radiation ISW effect contributes significantly to intermediate angle anisotropies. (b) However, as already noted in Fig. 2, the late ISW effect appearing at the left is much more significant than the corresponding $\Lambda$ effect. Thus on all angular scales, the total ISW contribution dominates the SW effect. Contours curve away from the curvature scale $\log (k * \mathrm{Mpc})=-3.8$ due to suppression of the potentials from the Poisson equation. Scales depicted in Fig. $5 \mathrm{c}, \mathrm{d}$ are marked here with dashed lines.

Figure 7. Open and $\Lambda$ isocurvature photon spectrum $\left(\Omega_{0}=0.1, h=0.5\right.$, standard recombination). Unlike their adiabatic counterparts, the potential grows in the radiation domination era only to turn over and decay in the curvature and $\Lambda$ dominated era. The ISW contribution will thus smoothly match onto the SW contribution. This has the effect of merging the SW and ISW ridges to make a wide feature that contributes broadly in $\ell$. For $\Lambda$ models, the radiation ISW effect completely dominates over the $\Lambda$ ISW effect. Scales depicted in Fig. 8 are marked here in dahsed lines.

Figure 8. Analytic separation of isocurvature large angle anisotropies $\left(\Omega_{0}=0.1, h=0.5\right.$, standard recombination, arbitrary normalization). Scales are chosen to match the features in Fig. 7. In general, isocurvature models have strong early ISW contributions which mimic and coherently boost the SW effect. $\Lambda$ models: (a) Notice that the shape of the SW and ISW effects are identical at large scales. (b) Even at the late ISW peak, the early ISW contributions are so strong that the late contributions are never apparent unlike the adiabatic model. Open models: (c) As with $\Lambda$ models, radiation epoch contributions are significant making the SW and ISW contributions similar for large scales. (d) Near the peak of the curvature ISW contribution however, the relative contributions are similar to the adiabatic case.

Figure 9. The acoustic oscillations $\left(\Omega_{0}=0.2, h=0.5\right.$, no recombination). The photonbaryon fluid acts like an oscillator in a potential well. The dipole, i.e. the photon velocity $V_{\gamma}$, is increasingly suppressed with respect to the monopole as $(1+R)^{-1 / 2}$, where the $\sqrt{3}$ accounts for the three degrees of freedom in the dipole. Scales which reach an extrema in the monopole at last scattering will correspond to the so-called "Doppler peaks" in the anisotropy spectrum. Also displayed here is the semianalytic approximation described in the text, which is essentially exact. The small difference in the numerical amplitudes of $\Phi$ and $\Psi$ is due to the anisotropic stress of the neutrinos. Whereas the isocurvature case has $\Omega_{0}=\Omega_{b}$, the adiabatic model has $\Omega_{b}=0.06$ and a consequently smaller $R$.

Figure 10. Small scale isocurvature evolution and photon diffusion $\left(\Omega_{0}=0.2, h=0.5\right.$, no recombination). At small scales gravity may be ignored, yielding pure adiabatic oscillations. Perturbations in the photons damp once the diffusion length grows larger than the wavelength $k_{D}<k$. Likewise the adiabatic component of the baryon fluctuations also damps leaving them with the original entropy perturbation. After diffusion, the photons and baryons behave as separate fluids, allowing the baryons to grow once Compton drag becomes negligible $a>a_{d}$. Photon fluctuations are then regenerated by the Doppler effect as they diffuse across infalling baryons. The analytic approach for the photons in this limit apply between the drag epoch and last scattering $a_{d}<a<a_{*}$.

Figure 11. Angular scale of the "Doppler peaks" (standard recombination). The physical scale of the peaks is simply related to the sound horizon at last scattering. Peaks in the anisotropy today will correspond to multiples of the angle that this scale subtends on the sky $\ell_{p}=\pi r_{\theta} / r_{s}\left(\eta_{*}\right)$, as discussed in the text. Varying $\Lambda$ or $h$ increases both the sound horizon at $\eta_{*}$ and the present horizon $\eta_{0}$ leaving little effect. For open models, a given scale will correspond to a smaller angle by geodesic deviation. This simple analytic estimate for the peak location is valid for pure acoustic contributions and underestimates the scale of the first peak in low $\Omega_{0} h^{2}$ models due to neglect of the early ISW effect.

Figure 12. The total ISW effect $\left(\Omega_{0}=0.1, h=0.5\right.$, standard recombination, $k=k_{3} \times$ $\left.10^{-3} \mathrm{Mpc}^{-1}\right)$. (a) Adiabatic models. The decay of the potential as the scale enters the 
horizon due to pressure growth suppression causes the early ISW effect which boosts scales approaching the first acoustic oscillation. The largest scales which enter after radiation domination are boosted by the late ISW effects due to the rapid expansion in open and $\Lambda$ models, leaving a deficit at intermediate scales. (b) Isocurvature models. Scales which enter early during radiation domination do not grow as much due to the suppression in the potential. This enhances the large scale with respect to the small. Notice that the second adiabatic oscillation $\left(k_{3}=20\right)$ can be comparable to the first since the turnover in $\Phi$ occurs later. Only at the largest scales is the distinction between open and $\Lambda$ models manifest in the rms temperature fluctuations.

Figure 13. Open isocurvature baryon evolution under partial ionization $\left(\Omega_{0}=0.2, h=\right.$ 0.5). The baryons are released to grow in pressureless linear theory after Compton drag becomes negligible. Since this epoch becomes earlier as the ionization fraction is decreased, present day fluctuations will be larger for low $x_{e}$, if normalized to the ionization independent fluctuations at large scales. Unlike the CDM case, baryons have no potential wells into which they might fall after the drag epoch and the transfer function is extremely sensitive to the ionization history.

Figure 14. Open isocurvature baryon evolution in late reionization scenario $\left(\Omega_{0}=0.2, h=\right.$ $0.5)$. Here the universe is suddenly reionized to $x_{e}=1$ at redshift $z_{i}$ after a transparent period $1000>z>z_{i}$. Perturbations are released from drag following recombination only to suffer its effects once again between the ionization and drag epochs $z_{i}>z>z_{d}$. Thus the final fluctuations will be larger for later reionization.

Figure 15. Small scale isocurvature temperature evolution under partial reionization $\left(\Omega_{0}=\right.$ $0.2, h=0.5$, numerical). If the universe stays transparent after standard recombination at $z_{*} \simeq 1000$, the acoustic oscillations in the photon fluid will be frozen. However these large fluctuations are suppressed by diffusion damping in partially reionized models. Although the Doppler and other diffusive effects regenerate fluctuations at small scales, these effects are also suppressed under the diffusion length (i.e. the thickness of the last scattering surface).

Figure 16. The adiabatic matter transfer function $\left(h=0.5, \Omega_{b}=0.01\right)$. The transfer function has been scaled by $\left(\Omega_{0} h^{2}\right)^{-2}$ to compare different $\Omega_{0}$ values by requiring the same initial gravitational potential $\Phi$ (below the curvature scale). For scale invariant $n=1 \Lambda$ models, this normalization is equivalent to that determined by large scale anisotropies, since the SW effect dominates all but the lowest multipoles. Therefore the approximate relative amplitude of matter fluctuations can be directly read off from this plot. For open models, this is not true due to a more significant ISW effect and curvature effects at large scales which relate the potential to the initial power spectrum.

Figure 17. The isocurvature matter transfer function $\left(\Omega_{0}=0.2, h=0.5\right.$, numerical $)$. The baryon perturbations will have a prominent peak at the maximal Jeans scale since perturbations grow as $D(a)$ outside this scale, with a $k^{2}-3 K$ tail, and are suppressed inside it. The acoustic oscillations damp away in the highly ionized case since last scattering is delayed and the diffusion length grows. This leaves a flat small scale tail in the transfer function. Note also that in the low ionization scenarios, the Jeans length may not grown to its maximum matter dominated value by last scattering leading to a smaller scale for the peak. The growth suppression due to $\Lambda$ is less significant than that from curvature domination.

Figure 18. The full adiabatic photon power spectrum. The logarithmic contributions to the anisotropy in $\ell$ and $k$ [see equation (9)] are plotted here. Whereas in the $\Omega_{0}=1$ case only the SW ridge and acoustic peaks are prominent (top left and close up, top right), the $\Lambda$ and open cases show more complicated structure due to the ISW effect. Depending on the initial weightings, represented here as $\left|C_{A}\right|^{2} \propto \tilde{k}$, certain features may be emphasized over others. Notice the $\Lambda$ ISW effect at low $\ell$ and intermediate $k$ and the comparatively small open SW contribution at the foot of the ISW ridge. 
Figure 19. Large angle anisotropy dependence on the initial power spectrum $\left|C_{I}\right|^{2} \propto$ $\tilde{k}^{n}$ or $\left|C_{A}\right|^{2} \propto \tilde{k}^{n}$ for isocurvature and adiabatic scenarios respectively. $\left(\Omega_{0}=0.1, h=\right.$ 0.5 , standard recombination). Notice that for red spectra, geometric and/or cosmological constant effects play a role in determining the anisotropy whereas for very blue spectra, $\ell^{2} C_{\ell} \propto \ell^{2}$ for all models. Isocurvature models with $n \simeq-1$ to fit large scale structure will thus not be extremely sensitive to open or $\Lambda$ dominated universe effects. The normalization here is arbitrarily set at the quadrupole.

Figure 20. The full open isocurvature photon power spectrum for $\left|C_{I}\right|^{2} \propto \tilde{k}^{n}$. (a) Curvature scale weighted $n=-3$. The lack of a Poisson cut off in the isocurvature potential makes the nature of the open universe eigenfunctions apparent. The projection ridge crosses minimum eigenvalue $k=\sqrt{-K}$ (front edge) at roughly $\ell \sim 10$ corresponding to the fact that the lowest eigenfunction still has structure only around the curvature scale. This leads to the cutoff to low multipoles depicted in Fig. 19. (b) Small scale weighted $n=-1$. The main projection ridge does not dominate the anisotropy at the low order multipoles. Power from smaller physical scales (high $k$ ) bleeds in to boost the anisotropy. Thus anisotropies do not rise as rapidly with $\ell$ as predicted from the one to one conversion of $k$ onto $\ell$. For this model $n_{\text {eff }} \simeq 2$ at large angles.

Figure 21. Intermediate to small scale anisotropies. (a) Adiabatic models. Projection of the sound horizon at last scattering onto sky today determines the angular scale of the "Doppler peaks" (c.f. Fig. 11). The sound horizon is the same physical scale for open and $\Lambda$ models with fixed $\Omega_{0}$ but geodesic deviation makes it correspond to a smaller angle in the open case. Compared with the flat case, the $\Lambda$ model has a somewhat smaller angular scale due to the imperfect cancellation between the increase in the age of the universe today and at last scattering. (b) Isocurvature models. Anisotropies in the standard recombination scenario $\left(x_{e} \simeq 0\right.$ if $\left.z<1000\right)$ produce far to large fluctuations on the arcminute scale due to the steeply rising spectrum. Reionized models have their adiabatic fluctuations damped out by photon diffusion and a cancellation suppressed Doppler effect. Notice that large angle anisotropies are immune to ionization history effects for the open case but not for the $\Lambda$ case. This and the difference in the damping scale is mostly due to the projection effect.

Figure 22. Isocurvature temperature power spectrum. In this fully ionized $x_{e}=1$, low $\Omega_{0}=0.1 h=0.5$ model, the ISW effect makes a contribution equal to and with the same scale dependence as the cancelled Doppler (plus SW) term (DSW). The second order Vishniac term (V) dominates at small scales. The analytic approximation (solid) fails at large scales where cancellation arguments are not applicable.

Figure 23. Radial eigenfunctions of an open universe $X_{\nu}^{\ell}(\chi)$. (a) The isotropic $\ell=0$ function for several values of the wavenumber $\nu=\tilde{k} / \sqrt{-K}$. The zero crossing moves out to arbitrarily large scales as $\nu \rightarrow 0$, reflecting completeness. However, even as this "effective wavelength" becomes infinite, the function retains prominent structure only near the curvature scale $\chi$. A random superposition of these low $\nu$ modes cannot produce more than exponentially decaying structure larger than the curvature scale. (b) Low order multipoles in the asymptotic limit $\nu \rightarrow 0$. If most power lies on the curvature scale, the $\ell$-mode corresponding to the angle that the curvature radius subtends will dominate the anisotropy. The normalization is appropriate for comparing contributions to the anisotropy $\ell(2 \ell+1) C_{\ell} / 4 \pi$. Also shown is the location of the horizon $\chi=\eta_{0} \sqrt{-K}$ for several values of $\Omega_{0}$. If contributions to the anisotropy come from a sufficiently early epoch, the dominant $\ell$-mode will peak at this value. 
This figure "fig7.png" is available in "png" format from: http://arXiv.org/ps/astro-ph/9411008v1 
This figure "fig8.png" is available in "png" format from: http://arXiv.org/ps/astro-ph/9411008v1 
This figure "fig9.png" is available in "png" format from: http://arXiv.org/ps/astro-ph/9411008v1 
This figure "fig10.png" is available in "png" format from: http://arXiv.org/ps/astro-ph/9411008v1 
This figure "fig11.png" is available in "png" format from: http://arXiv.org/ps/astro-ph/9411008v1 
This figure "fig12.png" is available in "png" format from: http://arXiv.org/ps/astro-ph/9411008v1 
This figure "fig13.png" is available in "png" format from: http://arXiv.org/ps/astro-ph/9411008v1 
This figure "fig14.png" is available in "png" format from: http://arXiv.org/ps/astro-ph/9411008v1 
This figure "fig15.png" is available in "png" format from: http://arXiv.org/ps/astro-ph/9411008v1 
This figure "fig16.png" is available in "png" format from: http://arXiv.org/ps/astro-ph/9411008v1 
This figure "fig17.png" is available in "png" format from: http://arXiv.org/ps/astro-ph/9411008v1 
This figure "fig18.png" is available in "png" format from: http://arXiv.org/ps/astro-ph/9411008v1 
This figure "fig19.png" is available in "png" format from: http://arXiv.org/ps/astro-ph/9411008v1 
This figure "fig20.png" is available in "png" format from: http://arXiv.org/ps/astro-ph/9411008v1 
This figure "fig21.png" is available in "png" format from: http://arXiv.org/ps/astro-ph/9411008v1 
This figure "fig22.png" is available in "png" format from: http://arXiv.org/ps/astro-ph/9411008v1 
This figure "fig23.png" is available in "png" format from: http://arXiv.org/ps/astro-ph/9411008v1 